\title{
Overview of CSR codes
}

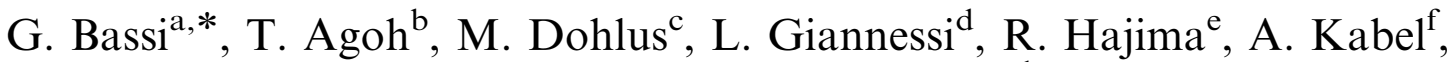 \\ T. Limberg ${ }^{\mathrm{c}}$, M. Quattromini ${ }^{\mathrm{d}}$ \\ ${ }^{a}$ Department of Mathematics and Statistics, UNM, Albuquerque, NM 87131, USA \\ ${ }^{\mathrm{b}}$ High Energy Accelerator Research Organization (KEK) Oho 1-1, Tsukuba, Ibaraki, Japan \\ ${ }^{\mathrm{c}}$ DESY, Hamburg, Germany \\ ${ }^{\mathrm{d}}$ Ente nazionale per le Nuove tecnologie, l'Energia e l'Ambiente, Divisione di Fisica Teorica e Matematica Applicata, C.R. Frascati, \\ Via E. Fermi 27, 00044 Frascati, Rome, Italy \\ e Japan Atomic Energy Research Institute, Tokai-mura, Ibaraki 319-1195, Japan \\ ${ }_{\mathrm{f}}^{\mathrm{f}}$ tanford Linear Accelerator Center, Stanford, CA, USA
}

Available online 18 November 2005

\begin{abstract}
Coherent synchrotron radiation (CSR) effects play an important role in accelerator physics. CSR effects can be negative (emittance growth in bunch compressors and microbunching instability) or positive (production of CSR in a controlled way). Moreover, CSR is of interest in other fields such as astrophysics. Only a few simple models have been solved analytically. This motivates the development of numerical procedures. In this review article we overview different numerical methods to study CSR effects.
\end{abstract}

(C) 2005 Elsevier B.V. All rights reserved.

PACS: 41.60.-m; 41.60.Ap; 41.75.-i; 41.75.Fr; 41.20.-q

Keywords: CSR; Coherent synchrotron; Bunch compressor; Charged-particle beams

\section{Introduction}

Coherent synchrotron radiation (CSR) is expected to play an important and often detrimental role in various advanced accelerator projects, for instance in linac-based coherent light sources [1] and energy recovery linacs [2]. A large concern is that CSR may cause transverse emittance growth in bunch compressors and a microbunching instability [3-7]. Besides negative effects, the interest for the utilization of high power radiation in the terahertz and far infrared region in the field of chemical, physical and biological processes has led several synchrotron facilities to test the possibility of producing CSR in a controlled way [8]. Another field of interest of CSR effects is astrophysics: the high brightness temperatures of radio emission of pulsars implies a coherent emission mechanism and some part of the radio emission of extragalactic jets may be coherent [9]. These arguments strongly motivate the

\footnotetext{
${ }^{*}$ Corresponding author.

E-mail address: gbassi@math.unm.edu (G. Bassi).
}

development of analytical and numerical tools to understand CSR. For an introduction and a comprehensive historical review of CSR see Ref. [10] and references therein. Analytical efforts have led to a considerable understanding of CSR in simple models, however for realistic models one has to rely on numerical studies. During the last decade several numerical methods have been developed to study CSR effects. Some of them are well known and others are under development. Agoh (Section 2), Bassi (Section 3), Giannessi and Quattromini (Section 4), Hajima (Section 5) and Kabel (Section 6) have written contributions for these proceedings and presented talks on CSR at the ERL2005 workshop. In addition, a contribution written by Dohlus and Limberg (Section 7) is included. In this introduction, we comment briefly on these contributions as well as give brief overviews of the codes developed by Borland, Emma, Rui Li and Warnock.

The method developed by Agoh (Section 2) is based on the assumptions that the pipe radius $a$ is much smaller then the bending radius $\rho$, that the field components propagating backward can be ignored (paraxial approximation) and 
that the bunch is rigid. To remove the last assumption, a technique of Gaussian series expansion in a particle tracking simulation has been implemented. These assumptions allow Maxwell equations to be solved by a finite difference scheme. The field calculation uses a 1D Fourier transform and includes shielding by a beam pipe. The method has been tested against analytical models.

Bassi et al., (Section 3) have developed a new approach to study CSR from arbitrary planar orbits. It is based on a fully self-consistent Vlasov-Maxwell treatment. The main motivation is that the Vlasov approach will have less noise than the standard particle approaches and will be able to follow densities. In a first attempt, the method was implemented in a full Fourier domain. The idea, due to Warnock, was to apply the method of stationary phase to get an efficient evaluation of the fields. A summary of this attempt can be found in Ref. [11], where the conclusion is that the method is only partially effective. The current approach works in a 1D Fourier domain. The field calculation, which is very delicate and is the most time consuming part of the algorithm, is done in the lab frame while the Vlasov equation is integrated in the beam frame.

Giannessi and Quattromini have developed a fully 3D Monte Carlo code called TREDI (Section 4) devoted to simulations of charged beam dynamics by direct integration of particle trajectories, accounting for self-fields through Lienard-Wiechert potentials. The code uses extended macroparticles with a form factor and a smoothing approach to regularize the divergences of electromagnetic fields.

Hajima (Section 5) has a simple analytical model to estimate the emittance growth due to the shielded CSR through an ERL loop. The emittance along the beam path has been compared with the code elegant and the emittance compensation by cell-to-cell phase matching is confirmed.

The code TraFiC ${ }^{4}$ (Section 6), developed by Kabel et al., models the self-interaction of the bunch by storing away the complete history of a bunch of extended macroparticles with Gaussian profiles whose centroids are tracked. Electric and magnetic fields at any given point can be calculated by integrating their profile over a retarded time interval. The momentum kick on each particle originates from the sum of the fields of all macroparticles, making TraFiC $O\left(N^{2}\right)$, where $N$ is the number of macroparticles. For self-consistent calculations TraFiC ${ }^{4}$ has two different algorithms. Among other features, $\mathrm{TraFiC}^{4}$ has the possibility to calculate the fields due to CSR at observation points far away from the bunch.

The code CSRtrack (Section 7), developed by Dohlus and Limberg, is a new code for the simulation of CSR effects on the beam dynamics of linear accelerators. It offers different models to calculate CSR effects. On one side the fast and efficient $1 \mathrm{D}$ method is available (as in Borland's and Emma's) program, on the other hand subbunch computation similar to TraFiC ${ }^{4}$ can be done. CSRtrack and TraFiC ${ }^{4}$ use the same solver for the calculation of sub-bunch fields. Here we summarize some common features and some differences between CSRtrack and TraFiC ${ }^{4}$ :

(1) $\mathrm{TraFiC}^{4}+$ CSRtrack: shielding by conducting planes; mirror charge approach;

(2) CSTrack does not store the complete history; it estimates the "history" or the position of particles/ sub-bunches in the past by "back-tracking";

(3) the effort for the field calculation can be reduced by meshing techniques:

(a) "pseudo Green's function method": meshing of the field of a sub-bunch;

(b) meshing of the EM-field of the complete distribution;

therefore, the effort can be reduced to $O(N)$.

The program elegant [12] is a general-purpose accelerator simulation code that incorporates a 1D CSR algorithm for dipoles and drift spaces. Originally [13], the algorithm was based on a formula [14] for the energy change by a line-charge distribution in a dipole magnet, with extensions for the energy change in downstream drift spaces [13]. Although these extensions were not derived in a rigorous way, arguments for their plausibility are given in Ref. [13]. Versions 14.5 and later of elegant allow use of either the original treatments or a rigorous treatment of the drift space effects [15]. There is some experimental evidence [16] that the original models are better, in spite of not having a rigorous footing. In these algorithms, retardation effects are not properly included in the sense that CSR effects are computed from the projected line charge distribution at the present time, rather than from the true history of the distribution. The advantage of the code is that it is very fast and allows integrated simulation of a linear accelerator, transport line, or storage ring with CSR effects, a 1D longitudinal space charge (LSC) model, transverse and longitudinal wakefields, and six-dimensional beam transport. The high longitudinal resolution made possible by the use of a 1D model led to discovery of the CSR-driven microbunching instability in the LCLS [3], the first indication of such an instability in a bunch compression system. It has also permitted exploration of microbunching instabilities involving CSR, LSC, and longitudinal wakefields [6,7], along with possible cures such as strong wigglers or laser/undulator beam heaters.

The code by Emma [15] uses results for the CSR wake in a short magnet [14] and is based on expressions for the wake in the limit of very large values of the relativistic factor $\gamma, \gamma \rightarrow \infty$, for both the entrance and the exit of the magnet. Like elegant, the field calculation is 1D. The code implements a subset of the CSR algorithms available in elegant and was used first to do simulations with the new drift space model discussed in Ref. [15]. For an application of the code to the study of CSR effects in a chicane bunch compressor see Ref. [17]. For a comparison 
of the code (together with the code elegant) with an analytical model to study microbunching instability see Ref. [7].

Rui $\mathrm{Li}$ has developed a 2D code [18] based on the calculation of retarded potentials where the bunch distribution is simulated by a set of Gaussian discs with zero vertical size. The bunch with total charge $Q$ is simulated by $N_{\mathrm{m}}$ number of macroparticles, where each macroparticle has charge $q_{\mathrm{m}}=Q / N_{\mathrm{m}}$ and rms size $\sigma_{\mathrm{m}}$. For a macroparticle with centroid at $\mathbf{r}_{0}(t)=\left(x_{0}(t), y_{0}(t)\right)$ at time $t$, its charge distribution is given by $\rho_{\mathrm{m}}(\mathbf{r}, t)=q_{\mathrm{m}} n_{\mathrm{m}}\left(\mathbf{r}-\mathbf{r}_{0}(t)\right)$, where $n_{\mathrm{m}}$ is a Gaussian with variance $\sigma_{\mathrm{m}}^{2}$.

In addition to initiating the work on the self consistent Vlasov-Maxwell approach mentioned above Warnock et al. have developed a Vlasov approach to CSR using a CSR impedance formalism [19-21]. The calculation is based on a time domain integration of the nonlinear Vlasov equation and is detailed enough to study microwave instabilities.

Most of the above codes have been tested against a benchmark bunch compressor [22].

\section{Calculation of coherent synchrotron radiation by paraxial approximation-T. Agoh}

We have developed a method to compute CSR using a grid. The electromagnetic field is handled in the frequency domain under a boundary condition of the vacuum chamber. In our method shielding effect by a beam pipe can be considered in a transient state. It is important to investigate the CSR effect in ERLs (Energy Recovery Linac) where the low energy beam is injected to the ring, we show a method of CSR simulation for low energy electrons.

\subsection{Introduction}

In the spectrum of synchrotron radiation, the component such that the wavelength is longer than the bunch length is coherent and called CSR. When a bunch is very short, it emits CSR which is intense and harmful for beams. Usually, it is hard to simulate CSR using a grid, because the wavelength is short, and the simulation requires a large number of grid points. But we make it possible with the paraxial approximation. Using our method, we can consider a pipe-shaped chamber by solving a fundamental equation.

\subsection{Theory}

We use the following assumption:

(a) $\varepsilon \equiv \sqrt{a / \rho} \ll 1$ : the pipe radius $a$ is much smaller than the bending radius $\rho$.

(b) The field components propagating backward are ignored (paraxial approximation).

(c) The bunch is rigid. But a macroparticle tracking simulation can remove the assumption (c).
From Maxwell equations,

$\boldsymbol{\nabla}(\boldsymbol{\nabla} \cdot \check{\mathbf{E}})-\boldsymbol{\nabla} \times(\boldsymbol{\nabla} \times \check{\mathbf{E}})-\frac{\partial^{2} \check{\mathbf{E}}}{\partial t^{2}}=\mu_{0}\left(\boldsymbol{\nabla} \check{J}_{0}+\frac{\partial \check{\boldsymbol{J}}}{\partial t}\right)$

where the variables having the symbol ${ }^{-}$are in the time domain. $\breve{J}_{0}, \breve{\boldsymbol{J}}$ are the charge density and the current, $\mu_{0}$ is the permeability of the vacuum. We put the speed of light equal to one $(c=1)$. We employ the coordinate system where $s$ is the length along the reference orbit, $x$ (horizontal), $y$ (vertical) are perpendicular to $s$. We assume that the current goes along the $s$-axis: $\boldsymbol{J}=J_{0} \boldsymbol{e}_{s}$. We Fourier transform Eq. (2.1) via

$\check{\mathbf{E}}(x, y, z ; s) \propto \mathbf{E}(x, y, k ; s) \mathrm{e}^{\mathrm{i} k z}$

with $z \equiv s-t$. $k$ is the wave number of the field. Ignoring the small terms $O\left(\varepsilon^{2}\right)$, and also the term of second derivative with respect to $s: \partial^{2} \boldsymbol{E}_{\perp} / \partial s^{2}$ (paraxial approximation), we obtain the fundamental equation of the transverse fields in the ultrarelativistic limit

$\frac{\partial \boldsymbol{E}_{\perp}}{\partial s}=\frac{\mathrm{i}}{2 k}\left[\left(\boldsymbol{\nabla}_{\perp}^{2}+\frac{2 k^{2} x}{\rho}\right) \boldsymbol{E}_{\perp}-\mu_{0} \boldsymbol{\nabla}_{\perp} J_{0}\right]$

where $\boldsymbol{\nabla}_{\perp}=\left(\partial_{x}, \partial_{y}\right)$ and $\nabla_{\perp}^{2}=\partial_{x}^{2}+\partial_{y}^{2}$. The longitudinal field in the $k$-domain is given by

$E_{s}=\frac{\mathrm{i}}{k}\left(\frac{\partial E_{x}}{\partial x}+\frac{\partial E_{y}}{\partial y}-\mu_{0} J_{0}\right)$.

Considering a moderate energy, i.e., a finite Lorentz factor $\gamma$, if we define a Fourier transform by Eq. (2.2) with $z \equiv s-v t$, we can get the fundamental equation

$\frac{\partial \boldsymbol{E}_{\perp}}{\partial s}=\frac{\mathrm{i}}{2 k}\left[\left(\boldsymbol{\nabla}_{\perp}^{2}+2 k^{2}\left(\frac{x}{\rho}-\frac{1}{2 \gamma^{2}}\right)\right) \boldsymbol{E}_{\perp}-\mu_{0} \boldsymbol{\nabla}_{\perp} J_{0}\right]$.

Assuming a stationary field without source, Eq. (2.5) reduces to Eq. (10) of Ref. [23]. Eq. (2.5) can be solved using the same algorithm as Eq. (2.3), (not yet performed). However, ignoring the term $2 \mathrm{i} k E_{s} / \rho$ which appears from the first term of Eq. (2.1) in the frequency domain, gives a relative error $\Delta$ :

$\Delta=\frac{2 \mathrm{i} k}{\rho} E_{s} / 2 k^{2} \frac{x}{\rho} E_{x} \sim \frac{\sigma_{z}}{a} \frac{1}{\gamma}$

where $\sigma_{z}, a$ are the bunch length and the pipe radius. For example, the relative error (2.6) is $\Delta=10^{-3}$ for $\sigma_{z}=1 \mathrm{ps,}$ $a=3 \mathrm{~cm}, \gamma=10$. That is, application of Eq. (2.5) is limited to about $5 \mathrm{MeV}$ for these parameters. In the following Eq. (2.3) is used and not Eq. (2.5).

\subsection{Algorithm}

Grid size can be large in the paraxial approximation. However, the transverse beam size is much smaller than the pipe size, so a fine grid is required to resolve the transverse charge distribution of $J_{0}$ in Eq. (2.3). We can solve the problem by removing the source term $J_{0}$. The field is separated as $\mathbf{E}=\mathbf{E}^{r}+\mathbf{E}^{b}$ where $\mathbf{E}^{r}, \mathbf{E}^{b}$ are the radiation field and the beam field. $\mathbf{E}^{b}$ is defined by the field in a drift 
space that satisfies

$\boldsymbol{\nabla}_{\perp}^{2} \mathbf{E}_{\perp}^{b}=\mu_{0} \boldsymbol{\nabla}_{\perp} J_{0}$

With Eq. (2.7), the term $\mu_{0} \nabla J_{0}$ can be replaced with the known field $\mathbf{E}_{\perp}^{b}$. For example, in the case of a bunch with zero transverse beam size, $\mathbf{E}_{\perp}^{b}$ is given by

$\mathbf{E}_{\perp}^{b}=\frac{\mu_{0} \lambda}{2 \pi} \frac{1}{x^{2}+y^{2}}\left(\begin{array}{l}x \\ y\end{array}\right)$

where $\lambda$ is the longitudinal distribution of the bunch. For a bunch which has a Gaussian distribution in the transverse direction, the beam field is given by Bassetti-Erskine formula [25]. But if the transverse beam size is much smaller than the typical scale length of CSR field, the longitudinal field of CSR does not depend on the transverse beam size. So usually, Eq. (2.8) is enough to investigate the longitudinal effects. With the beam field, Eq. (2.3) becomes

$\frac{\partial \boldsymbol{E}_{\perp}^{r}}{\partial s}=\frac{\mathrm{i}}{2 k}\left[\left(\boldsymbol{\nabla}_{\perp}^{2}+\frac{2 k^{2} x}{\rho}\right) \boldsymbol{E}_{\perp}^{r}+\frac{2 k^{2} x}{\rho} \mathbf{E}_{\perp}^{b}\right]$.

Using the leap-frog method, $\boldsymbol{E}_{\perp}^{r}$ in Eq. (2.9) can be solved step by step with respect to $s$ as an initial value problem. The nature of the beam pipe gives the boundary condition, we can handle a resistive wall. After solving Eq. (2.9), adding the beam field to the radiation field gives the actual field. In case that we solve it with a rectangular grid $(\Delta x, \Delta y)$ in the transverse plane, von Neumann stability analysis imposes a condition on the grid size

$\frac{2 \Delta s}{k}\left(\frac{1}{\Delta x^{2}}+\frac{1}{\Delta y^{2}}\right)<1$.

Since we are working in the frequency domain, Eq. (2.9) will be solved for a rigid bunch. Still a technique of Gaussian series expansion in the particle tracking simulation enables us to consider the time evolution (sdependence) of the longitudinal bunch distribution. That is, at first we compute CSR for a Gaussian distribution $G_{i}$ with a small width $\sigma_{0}$

$\check{\lambda}(z) \equiv \sum_{i} G_{i}(z)$

$G_{i}(z) \equiv \frac{A_{i}}{\sqrt{2 \pi} \sigma_{0}} \mathrm{e}^{-\left(z-z_{i}\right)^{2} / 2 \sigma_{0}^{2}}$

By superposing $G_{i}$ with a proper height $A_{i}$ and spacing $z_{i}=i \Delta z$, as shown in Fig.1, we can calculate CSR for a bunch of any distribution. In other words, $G_{i}$ is a kind of Green function having a Gaussian distribution. Rigorously speaking, though this technique is not self-consistent, it is sufficient to calculate CSR for general bunch distributions. However, we have to mention also the limit of this technique. If the bunch has a fine structure such as microwave instability, the width of the Gaussian Green function $\sigma_{0}$ must be small enough to express the structure of the bunch. To do so, the tracking simulation requires a

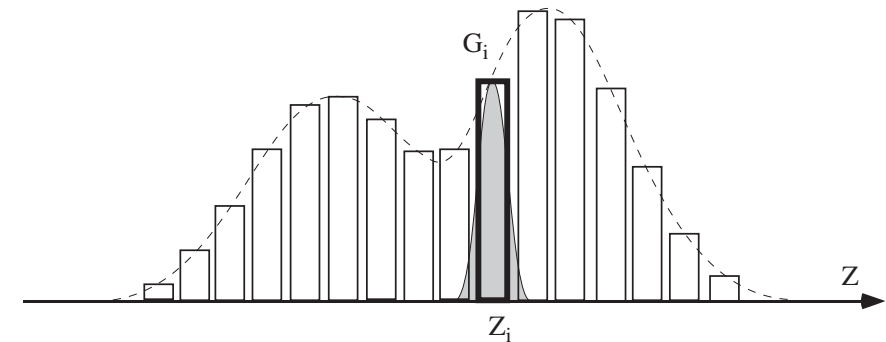

Fig. 1. Gaussian series expansion for arbitrary bunch distribution. The dashed curve is the longitudinal bunch distribution which consists of bins. A small Gaussian distribution with gray $G_{i}$ is placed for a bin of macroparticle distribution.

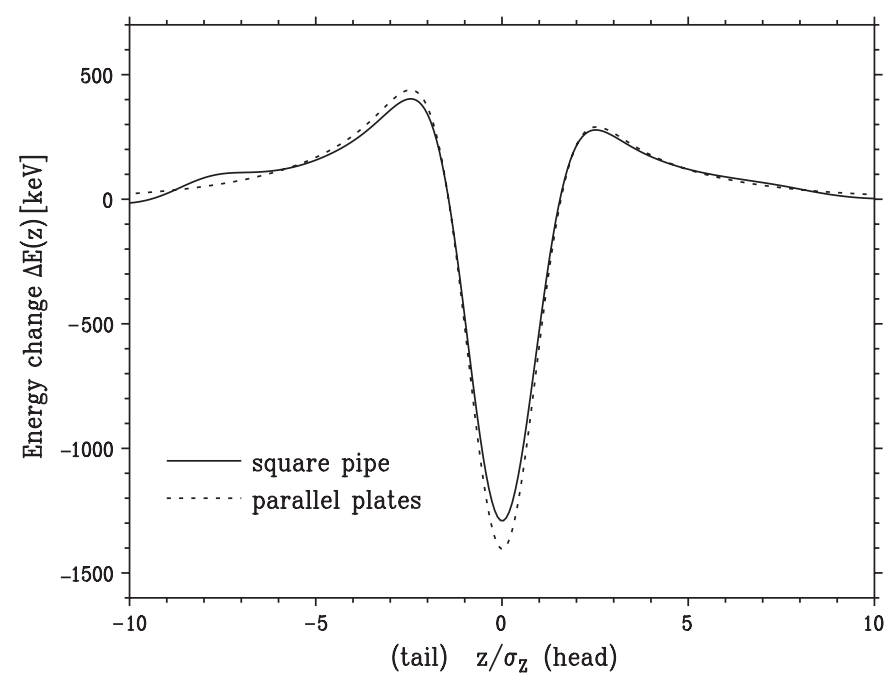

Fig. 2. Energy change due to CSR in a bending magnet. The horizontal axis is the longitudinal position in the bunch. The bunch length is $\sigma_{z}=1 \mathrm{ps}$, the bending radius is $16.3 \mathrm{~m}$, the vacuum chamber has a square cross section (solid line) and parallel plates (dotted line) with a full height of $94 \mathrm{~mm}$

lot of macroparticles to suppress the statistical fluctuation; such a simulation tends to be expensive. Though the limit of the expression is given by the computer's specification, we usually use one million macroparticles on an ordinary PC. However, even one million macroparticles would not be enough to express microwave instability which induces a very tiny structure in the bunch.

\subsection{Shielding by a square pipe}

An example of the energy change due to CSR in a single bend is shown in Fig. 2 where it is assumed $\gamma=\infty$. The bunch length is $1 \mathrm{ps}$. The difference between square pipe (solid) and parallel plates (dashed) is about 9\% around the center of the bunch. When the bunch length is $10 \mathrm{ps}$, the energy change is shown in Fig. 3. In this case, shielding is very strong, also side walls greatly contribute to the shielding effect. The difference between square pipe and parallel plates amounts to $46 \%$. 


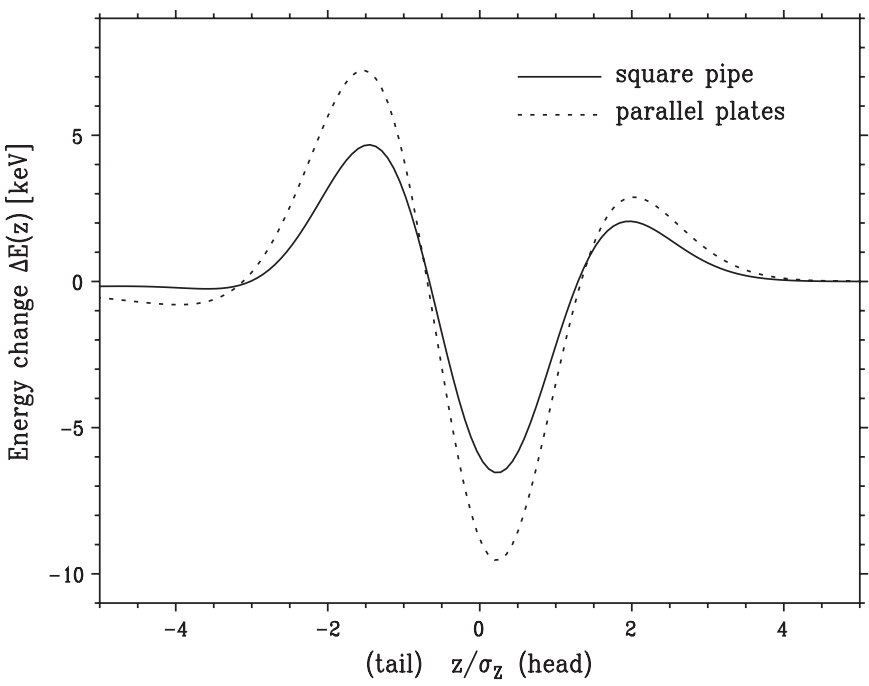

Fig. 3. Energy change due to CSR in a bending magnet. The bunch length is $\sigma_{z}=10 \mathrm{ps}$, the other parameters are the same as Fig. 2 .

\subsection{Conclusion}

We have performed a simulation of CSR using a grid. Owing to the paraxial approximation, the computing cost is small, and we can simulate it on a personal computer. Our method is conceived to work not only in the ultrarelativistic limit but also for a moderate energy, though we have not yet performed it. The lower limit of energy may be around $5 \mathrm{MeV}$. Another advantage of our method is that shielding by a beam pipe can be properly considered. When the bunch length is $1 \mathrm{ps}$, the parallel plates approximation yields error of $9 \%$ in the particle's energy change. So, if the vacuum chamber has a cross section whose height/width ratio is close to one like a square or round pipe, a proper model of the chamber is needed [24].

\subsection{Acknowledgments}

I would like to thank E. Forest and K. Yokoya for many useful comments. I also wish to thank G. Bassi for his organization.

\section{A Vlasov treatment of coherent synchrotron radiation from arbitrary planar orbits-G. Bassi}

We are working on a new approach to study CSR from arbitrary planar orbits [26,27]. It is based on a fully selfconsistent Vlasov-Maxwell (VM) treatment. The Vlasov approach will have less noise than the standard particle approaches and will be able to follow densities. However, it is computationally intensive and it is necessary to combine analytical and computational techniques which are accurate and yet give us a fast method. Our progress is given in Refs. [26] and [27], in this paper we give details of our algorithm. We first discuss the VM model followed by details of the field computation which is the most time consuming part of our algorithm. The field calculation is done in the lab frame, however we integrate the Vlasov equation in the beam frame and so we then discuss the beam frame coordinates, the beam to lab transformation and the combined VM integration. The computation is quite delicate and we end by discussing some of the numerical issues we have faced so far.

\subsection{Statement of the problem}

We consider the case where guiding dipole fields give planar motion in a $(Z, X)$ plane and we ignore CSR forces in the $Y$-direction perpendicular to the $(Z, X)$ plane. We include vacuum chamber shielding modeled by two perfectly conducting plates at $Y= \pm g$. The relevant CSR fields are $E_{Z}, E_{X}$ and $B=B_{Y}$ and are governed by the boundary value problem for the 2-D wave equation

$\left(\partial_{Z}^{2}+\partial_{X}^{2}-\partial_{u}^{2}\right) \mathscr{E}=H(Y) \mathscr{P}, \mathscr{E}(\mathbf{R}, Y= \pm g, u)=0$.

Here $\mathscr{E}(\mathbf{R}, Y, u)=\left(E_{Z}, E_{X}, B\right), \mathbf{R}=(Z, X), \mathscr{S}=\left(\nabla \rho_{\mathrm{L}} /\right.$ $\left.\varepsilon_{0}+\mu_{0} c \partial \mathbf{J}_{\mathrm{L}} / \partial u,-\mu_{0}\left(\nabla \times \mathbf{J}_{\mathrm{L}}\right)_{Y}\right), H(Y)$ is an arbitrary fixed vertical distribution of charge with $\int H=1, u=c t$ and $\rho_{\mathrm{L}}(\mathbf{R}, u), \mathbf{J}_{\mathbf{L}}(\mathbf{R}, u)$ are the charge/current densities in the lab frame.

The particles evolve by the Lorentz equation

$\frac{\mathrm{d}}{\mathrm{d} u} m \gamma \mathbf{V}=\frac{q}{c}\left(\mathbf{E}+\mathbf{V} \times\left(B+B_{\mathrm{e}}\right) \mathbf{e}_{Y}\right)$

where $\mathbf{V}=\left(V_{Z}, V_{X}\right), B_{\mathrm{e}}$ is the external dipole field and $\mathbf{e}_{Y}$ is the unit vector in the $Y$ direction, $(Z, X, Y)$ being a righthanded system. Using the fact that $\dot{\gamma}=\left(q / m c^{2}\right) \mathbf{E} \cdot \mathbf{V}$, the Lorentz equation can be written in system form as

$$
\begin{aligned}
\dot{\mathbf{R}}= & \frac{\mathbf{V}}{c} \\
\dot{\mathbf{V}}= & \frac{q}{m c \gamma(V)}[\mathbf{E}(\mathbf{R}, Y, u)+\mathbf{V} \times(B(\mathbf{R}, Y, u) \\
& \left.\left.+B_{\mathrm{e}}(\mathbf{R})\right) \mathbf{e}_{Y}-\frac{1}{c^{2}}(\mathbf{E}(\mathbf{R}, Y, u) \cdot \mathbf{V}) \mathbf{V}\right]
\end{aligned}
$$

and the Vlasov equation for the phase space density $F=$ $F(\mathbf{R}, \mathbf{V}, u)$ becomes

$$
\frac{\partial F}{\partial u}+\dot{Z} \frac{\partial F}{\partial Z}+\dot{V}_{Z} \frac{\partial F}{\partial V_{Z}}+\dot{X} \frac{\partial F}{\partial X}+\dot{V}_{X} \frac{\partial F}{\partial V_{X}}=0 .
$$

Here $\cdot \equiv \frac{\mathrm{d}}{\mathrm{d} u}$. Eqs. (3.1) and (3.4), depend on $Y$ as a parameter only. In our basic VM model we use fields averaged over $Y$ in (3.4), i.e.,

$\mathscr{E}(\mathbf{R}, u):=\langle\mathscr{E}(\mathbf{R}, \cdot, u)\rangle=\int_{-g}^{g} H(Y) \mathscr{E}(\mathbf{R}, Y, u) \mathrm{d} Y$.

The averaged field can be computed much more quickly, and we believe that it will produce nearly the same dynamics in the $(Z, X)$ plane as the full field. 


\subsection{Field calculation}

After imposing boundary conditions at the parallel plates by the method of images the $Y$-average produces just a two-dimensional integral,

$$
\begin{aligned}
\mathscr{E}(\mathbf{R}, u)= & -\frac{1}{4 \pi} \sum_{k=-\infty}^{\infty}(-1)^{k} \int \mathrm{d} \mathbf{R}^{\prime} \\
& \times \frac{\mathscr{S}\left(\mathbf{R}^{\prime}, u-\left[\left(\mathbf{R}^{\prime}-\mathbf{R}\right)^{2}+(k h)^{2}\right)\right]^{1 / 2}}{\left.\left[\left(\mathbf{R}^{\prime}-\mathbf{R}\right)^{2}+(k h)^{2}\right)\right]^{1 / 2}} .
\end{aligned}
$$

where $h=2 g$. The integration in Eq. (3.5) is restricted to a very small part of the full $\mathbf{R}^{\prime}$ plane, because of the small size of the bunch, but it is awkward to locate this region owing to the fact that spatial and temporal arguments of the source both depend on $\mathbf{R}^{\prime}$. The task of integration is made drastically easier if we take the temporal argument to be a new variable of integration. We first go into polar coordinates $(\zeta, \theta)$, then use the temporal argument $v$ in place of the radial coordinate $\zeta$. That is,

$\mathbf{R}^{\prime}-\mathbf{R}=\zeta \mathbf{e}(\theta), \quad \mathbf{e}(\theta)=(\cos \theta, \sin \theta)$,

$v=u-\left[\zeta^{2}+(k h)^{2}\right]^{1 / 2}$.

This incidentally gets rid of the small divisor in Eq. (3.5), giving the field simply as an integral over the source

$\mathscr{E}(\mathbf{R}, u)=-\frac{1}{2 \pi} \sum_{k=0}^{\infty} a_{k} \int_{-\infty}^{u-k h} \mathrm{~d} v \int_{-\pi}^{\pi} \mathrm{d} \theta \mathscr{S}(\hat{\mathbf{R}}, v, k)$,

where $\quad \hat{\mathbf{R}}=\mathbf{R}+\sqrt{(u-v)^{2}-(k h)^{2}} \mathbf{e}(\theta)$

and $a_{k}=(-1)^{k}\left(1-\delta_{k 0} / 2\right)$. The derivation is presented in Ref. [26]. However, in Ref. [26] we did an integration by parts to get rid of the time derivative of the current density. We are investigating the relative merits of the two representations.

\subsection{Beam frame Vlasov equation}

The beam frame coordinates are defined in terms of a reference orbit $\mathbf{R}_{0}(s)=\left(Z_{0}(s), X_{0}(s)\right)$ where $s$ is arc-length and a reference particle travels on this orbit with constant speed $\beta c$ so its trajectory is $\mathbf{R}_{0}(\beta u)$. Thus a lab frame point $(Z, X)$ can also be specified in terms of Frenet-Serret

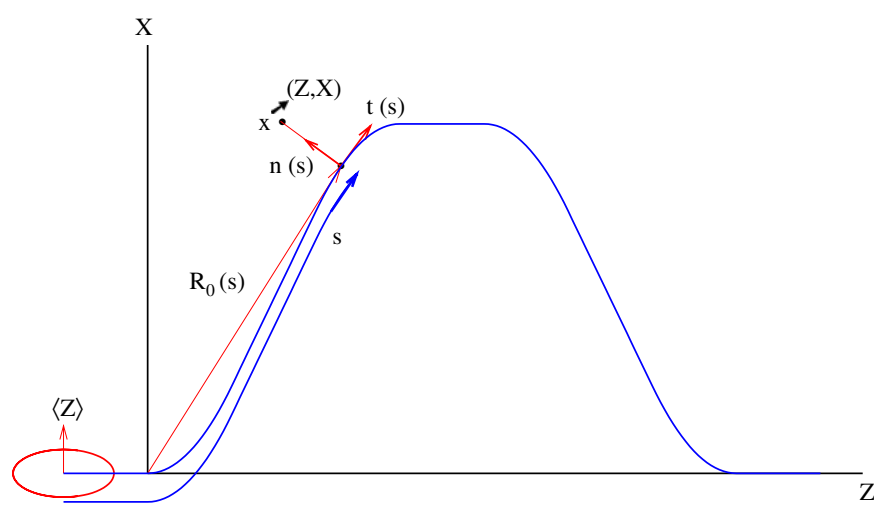

Fig. 4. Lab frame $(Z, X)$ and beam frame $(s, x)$ for a chicane bunch compressor. coordinates relative to the orbit by $\mathbf{R}=\mathbf{R}_{0}(s)+x \mathbf{n}(s)$ where $\mathbf{n}(s)=\left(-X_{0}^{\prime}(s), Z_{0}^{\prime}(s)\right)$ is the unit normal vector. The corresponding unit tangent is $\mathbf{t}(s)=\mathbf{R}_{0}^{\prime}(s)=$ $\left(Z_{0}^{\prime}(s), X_{0}^{\prime}(s)\right)$. This set up is shown in Fig. 4. After a change of independent variable from $u=c t$ to $s$, a convenient set of dynamical variables for motion in horizontal planes consists of the "beam frame" phase space coordinates $(\mathbf{r}, \mathbf{p})$, where $\mathbf{r}=(z, x)$ and $\mathbf{p}=\left(p_{z}, p_{x}\right)$. Here $z(s)=s-\beta c t(s)$, where $t(s)$ is the time of arrival at arc-length $s$. The conjugate variable is the relative energy deviation $p_{z}(s)=\left(E(s)-E_{0}\right) / E_{0}$, with $E_{0}=m \gamma_{0} c^{2}$ the energy of the reference particle, and $p_{x}(s)=v_{x}(s) / \beta c$ where $v_{x}$ is the velocity component along $\mathbf{n}$.

The equations of motion in the beam frame are easily determined from (3.3) and are given approximately by

$z^{\prime}=-\kappa(s) x, \quad p_{z}^{\prime}=F_{z}, \quad x^{\prime}=p_{x}, \quad p_{x}^{\prime}=\kappa(s) p_{z}+F_{x}$.

Here $^{\prime} \equiv \mathrm{d} / \mathrm{d} s$ and the collective force is

$$
\begin{aligned}
F_{z}= & \frac{q}{\beta c E_{0}} \mathbf{E} \cdot \mathbf{V} \\
F_{x}= & \frac{q}{E_{0} \beta^{2}}\left[-X_{0}^{\prime}(s)\left(E_{Z}-V_{X} B\right)\right. \\
& \left.+Z_{0}^{\prime}(s)\left(E_{X}+V_{Z} B\right)-\frac{\beta p_{x}}{c} \mathbf{E} \cdot \mathbf{V}\right]
\end{aligned}
$$

where $\mathbf{V}=\beta c\left(\mathbf{t}(s)+p_{x} \mathbf{n}(s)\right), \quad \mathbf{E}=\left(E_{Z}, E_{X}\right) \quad$ and $B$ are evaluated at $\mathbf{R}=\mathbf{R}_{0}(s)+x \mathbf{n}(s)$ and $u=(s-z) / \beta$. The main approximation in obtaining Eq. (3.8) is a linearization and these are the equations of standard linear optics perturbed by the collective force from CSR. The unperturbed version of Eq. (3.8) with $F_{z}=F_{x}=0$ can be solved explicitly in terms of the lattice functions $D(s), D^{\prime}(s), R_{56}(s)$ as discussed in Ref. [28]. This gives the transport map $\Phi(s \mid 0)$ from $s=0$ to arbitrary $s$, with inverse $\Phi(0 \mid s)$.

The Vlasov equation in the beam frame is

$\frac{\partial f}{\partial s}+z^{\prime} \frac{\partial f}{\partial z}+p_{z}^{\prime} \frac{\partial f}{\partial p_{z}}+x^{\prime} \frac{\partial f}{\partial x}+p_{x}^{\prime} \frac{\partial f}{\partial p_{x}}=0$

where $f=f(\mathbf{r}, \mathbf{p}, s)$.

\subsection{Beam to lab frame transformation}

To solve the Maxwell equations in lab frame we must express the lab frame charge/current density in terms of the beam frame phase space density, $f(\mathbf{r}, \mathbf{p}, s)$. Define

$$
\begin{aligned}
& \rho(\mathbf{r}, s)=Q \int \mathrm{d} \mathbf{p} f(\mathbf{r}, \mathbf{p}, s) \\
& \tau(\mathbf{r}, s)=Q \int \mathrm{d} \mathbf{p} p_{x} f(\mathbf{r}, \mathbf{p}, s)
\end{aligned}
$$

where $Q$ is the total charge and $f$ has unit integral. To a good approximation the lab frame charge and current densities are

$\rho_{\mathrm{L}}(\mathbf{R}, Y, u)=H(Y) \rho(\mathbf{r}, \beta u)$ 
$\mathbf{J}_{\mathrm{L}}(\mathbf{R}, Y, u)=\beta c H(Y)[\rho(\mathbf{r}, \beta u) \mathbf{t}(\beta u+z)$

$$
+\tau(\mathbf{r}, \beta u) \mathbf{n}(\beta u+z)] \text {, }
$$

where $\mathbf{r}=M^{\mathrm{T}}(\beta u)\left(\mathbf{R}-\mathbf{R}_{0}(\beta u)\right)$ with $M=(\mathbf{t}, \mathbf{n})$. The derivation of Eq. (3.11) will be discussed elsewhere.

\subsection{Proposed algorithm}

To solve the Vlasov-Maxwell equations at position $\hat{s}$, from the history $\left(s_{0} \leqslant s \leqslant \hat{s}\right)$ of $\rho_{\mathrm{L}}$ and $\mathbf{J}_{\mathrm{L}}$ we calculate the self fields using Eq. (3.7).Then we integrate the Vlasov equation for a step $\Delta$ with the method of local characteristics freezing at $\hat{s}$ the self fields. This gives the phase space density $f$ at $s+\Delta$. To get $\rho_{\mathrm{L}}$ and $\mathbf{J}_{\mathrm{L}}$ at $s+\Delta$ we then apply Eqs. (3.10) and (3.11).

\subsection{Numerical issues so far}

We are currently developing and testing our method against a benchmark bunch compressor [22]. Our results are discussed in Refs. [26] and [27]. Here we point out some numerical issues we faced in the development of our algorithm. The field calculation requires a careful understanding of the localization of the source in $\theta$ for $v \ll u-k h$ and is the most time consuming part of our approach. Moreover, the numerical integration must be done with high accuracy. Our idea is to the integrate the Vlasov equation in interaction picture to isolate the CSR dynamics and work with a slowly varying grid. We have seen a strong correlation between the phase space variables that makes the definition of the grid a difficult task.

\subsection{Acnowledgments}

Support from DOE grants DE-AC02-76SF00515 and DE-FG02-99ER1104 is gratefully acknowledged.

\section{The code TREDI-L. Giannessi and M. Quattromini}

The Lienard-Wiechert potentials approach is well-suited for the description of both CSR and pure space-charge effects. The TREDI Monte-Carlo program is a numerical code devoted to the simulation of beam dynamics in a wide range of contexts in acceleration physics [34].

It is well known that the fields produced by a charge distribution may be separated in two terms: a velocity (Coulomb) and an acceleration term. These two contributions have a different scaling behavior with the beam energy. Even though it is customarily accepted that the two contributions play a role in different dynamical regimes, for the velocity fields being relevant at low energies during the first acceleration stages and the acceleration fields providing a significant contribution along curved trajectories at high energy, it must be noted that an intermediate regime exists where none of the two contributions can be neglected. The reason of this can be understood by analyzing the retarded condition at which both the acceleration term and the velocity term must be evaluated. Let us assume, for example, that two charged particles spaced by $500 \mu \mathrm{m}$ follow the same path through a bending magnet with a curvature radius of $2.4 \mathrm{~m}$ at $100 \mathrm{MeV}$. The retarded condition

$(x-r)^{2} \equiv\left(x_{0}-r_{0}\right)^{2}-(\vec{x}-\vec{r})^{2}=0$

where $x$ and $r$ represent the space-time positions of the "target" and "source" particles, respectively, defines the effective interaction distance of the two particles, as shown in Fig. 5 for this specific example. The distance suddenly drops by nearly two orders of magnitude while the particles pass through the magnet, and then grows linearly with the distance from the magnet itself.

According to Ref. [29], the energy considered in the previous example (for a $100 \mathrm{~A}$ bunch) corresponds approximately to the transition from the laminar (collective) motion to the emittance-dominated regime, where Coulomb forces can be neglected. It must be noted, however, that this estimate holds for a purely rectilinear motion. The plot in Fig. 5 indicates that whenever the beam is deflected the "effective" bunch length is suddenly reduced and the space-charge dominated regime may come into play again.

The growing interest in recirculated machines where the beam does not follow an almost straight path and the necessity to deal with the longitudinal phase space manipulation in order to increase the peak current and to shorten the bunch length makes the previous example not just an academic exercise. Codes aimed at describing these situation must have a series of characteristics that are common both to the codes devoted to the injectors simulations and to those specifically designed for the CSR self-interaction. These features are mainly

- the full inclusion of both space charge and CSR forces;

- the capability of dealing with a fully three-dimensional geometry;

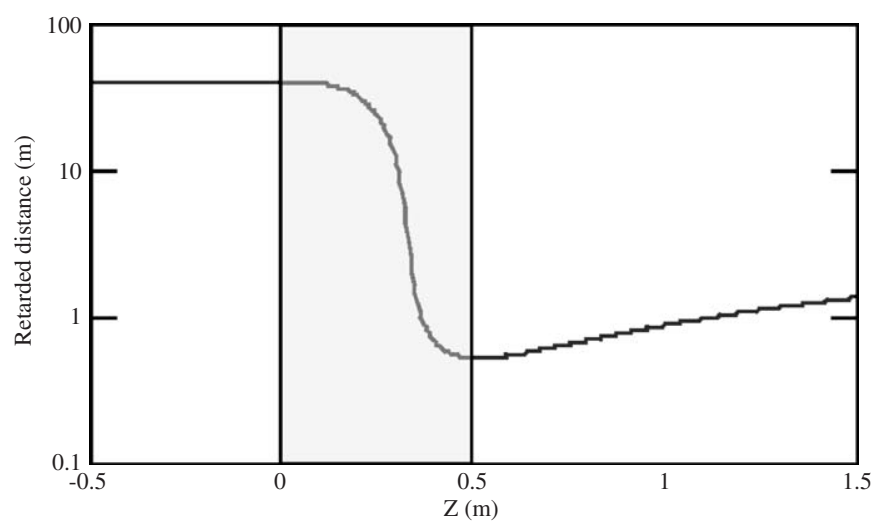

Fig. 5. Retarded distance between the two $100 \mathrm{MeV}$ charged particles spaced by $500 \mu \mathrm{m}$ while traversing a bending magnet of length $L=0.5 \mathrm{~m}$. The magnet is placed between $z=0$ and $z=0.5 \mathrm{~m}$. The curvature radius is $2.4 \mathrm{~m}$. 
- the description of relativistic dynamics even in the beam "rest" frame, in order to account for the large momentum spread in a bending.

The main feature of TREDI [30] is the implementation of the Lienard-Wiechert retarded potentials formalism for accounting of the self-fields effects in the beam dynamics in a three-dimensional geometry. TREDI is a particles pushing code, based on the solution of the Lorentz force equation for each simulated macroelectron. The electromagnetic (em) fields are the superposition of two terms: the external fields calculated from insertion devices as accelerating cavities or magnets, and the self-fields produced by the beam itself. This approach is efficient in the simulation of beam dynamics in three dimensions, due to the fact that the self-fields evaluation is obtained by means of the phase space particles coordinates, i.e. through the solution of ordinary differential equations, and are not the solution of partial differential equation (Maxwell's equations) requiring a set of constraints on a mesh size and step length to ensure numerical stability.

TREDI was initially developed with the purpose of simulating 3D effects associated to asymmetries in rf-guns, as cathode inhomogeneities [31] or multipolar effects in the acceleration fields. Subsequently, the code has been expanded and used also to model both CSR and spacecharge effects in magnetic chicanes [22]. At the present time the devices that can be included in the simulations are

- linac sections (both standing and traveling wave);

- solenoids;

- multipolar magnetic elements;

- dipoles and magnetic chicanes.

These elements can be placed in the simulation space with full control over position and orientation. The code can load directly the 2D or 3D maps used by Parmela [32] to describe the fields from external devices.

From the beginning of the development of TREDI much effort was devoted to solve the problem of EM self-fields regularization. This issue must be carefully addressed in all the models devoted to the solution of Maxwell-Lorentz equations based on a macro-particles approach. The singularity of the fields at the particles position must be regularized in order to produce consistent results. To this purpose, it can be shown that the decomposition of EM self-fields in velocity and acceleration terms, usually expressed in a nonmanifestly covariant form can be recast in terms of velocity and acceleration field strength tensors (which are relativistically covariant objects) that have specific features in terms of the regularization procedure. A covariant formulation presents two valuable properties:

(1) It is consistent and "general": the reduction of harmonic content of fields spectra turns to be naturally independent from the dynamical regime of the beam.
(2) The calculation can be actually carried out wherever the dynamical variables of the particle are directly available, namely the laboratory reference frame or the "beam" frame.

The code has been benchmarked in the simulation of RF injector dynamics [33] and despite the very different approach used, it exhibited a good agreement with other well established codes as Parmela [32]. The code has been implemented in the start to end simulation of a low energy test FEL experiment, where the FEL dynamics was accounted for self consistently [35]. This exercise was affected by a series of flaws mainly associated to the low number of macroparticles involved in the simulation. Its main purpose was just to show the code capabilities of simulating microbunching associated to radiative effects and by no means imply TREDI to be usable as a FEL simulation tool. A comparison of CSR effects simulations in a magnetic compressor with other codes described in this review can be found at Ref. [22] and up-to-date results are reported in Ref. [30]. TREDI has been utilized also in the simulation of diamagnetic field effects associated to space charge forces in the propagation in magnetic undulators [36].

\subsection{Acknowledgment}

The authors wish to thank Luca Serafini for stimulating the development of TREDI at its early stage.

\section{CSR analysis by first-order matrix-R. Hajima}

The emittance growth due to the CSR through an ERL loop can be estimated by first-order matrices of beam motion [37,38]. The matrix method is useful for optimizing an ERL loop, because it is much faster than particle tracking simulations.

A first-order equation of electron motion in a uniform field of a dipole magnet is

$x^{\prime \prime}=-\frac{x}{\rho^{2}}+\frac{1}{\rho}\left(\delta_{0}+\delta_{\mathrm{CSR}}+\kappa\left[s-s_{0}\right]\right)$

where $\rho$ is a bending radius, $\delta_{0}$ is initial momentum deviation normalized by reference momentum. The last two terms on the right-hand side are related to the CSR effect: $s$ is a coordinate along the ideal path, $s=s_{0}$ is the magnet entrance, $\delta_{\mathrm{CSR}}$ is normalized momentum deviation caused by CSR in the upstream path $\left(0<s<s_{0}\right), \kappa$ is CSR wake potential in the bending path normalized by the reference energy.

The transient CSR at the entrance and the exit of bending magnets has only small effects in a several-GeV ERL with a few picosecond bunch. In this constant CSR wake regime, Eq. (5.1) can be solved analytically, and electron dynamics in the bending plane can be tracked using $5 \times 5$ matrices

$\mathbf{x} \equiv\left(\begin{array}{lllll}x & x^{\prime} & \delta_{0} & \delta_{\mathrm{CSR}} & \kappa\end{array}\right)^{\mathrm{T}}$, 
$\mathbf{x}\left(s_{1}\right)=R_{0 \rightarrow 1} \mathbf{x}\left(s_{0}\right)$,

and the displacement of bunch slices in the $\left(x, x^{\prime}\right)$ phase space is expressed by CSR-wake dispersion function $\left(\zeta_{x}, \zeta_{x}^{\prime}\right)$.

$$
\begin{aligned}
& \left(\zeta_{x}\left(s_{1}\right) \zeta_{x}^{\prime}\left(s_{1}\right) 0 L_{b}\left(s_{1}\right) 1\right)^{\mathrm{T}} \\
& =R_{0 \rightarrow 1}\left(\zeta_{x}\left(s_{0}\right) \quad \zeta_{x}^{\prime}\left(s_{0}\right) \quad 0 \quad L_{b}\left(s_{0}\right) \quad 1\right)^{\mathrm{T}},
\end{aligned}
$$

where $L_{b}\left(s_{1}\right)$ is the total bending path length for $0<s<s_{1}$. A matrix for a sector dipole magnet is obtained by Green's function method

$$
\begin{gathered}
R_{\text {bend }}=\left(\begin{array}{ccc}
\cos \theta & \rho \sin \theta & \rho(1-\cos \theta) \\
-\frac{1}{\rho} \sin \theta & \cos \theta & \sin \theta \\
0 & 0 & 1 \\
0 & 0 & 0 \\
0 & 0 & 0 \\
\rho(1-\cos \theta) & \rho^{2}(\theta-\sin \theta) \\
\sin \theta & \rho(1-\cos \theta) \\
0 & 0 \\
1 & \rho \theta \\
0 & 1
\end{array}\right)
\end{gathered}
$$

Matrices for a quadrupole magnet and a drift space are obtained in a similar manner.

The emittance along a beam path is given by

$$
\varepsilon^{2}=\left(\varepsilon_{0} \beta_{x}+D^{2}\right)\left(\varepsilon_{0} \gamma_{x}+D^{\prime 2}\right)-\left(\varepsilon_{0} \alpha_{x}-D D^{\prime}\right)^{2}
$$

where $\left(\alpha_{x}, \beta_{x}, \gamma_{x}\right)$ are Courant-Snyder parameters, $\varepsilon_{0}$ is initial emittance and $\left(D, D^{\prime}\right)$ is rms spread of CSR wake dispersion defined as

$$
\left(D, D^{\prime}\right) \equiv \frac{\Delta E_{\mathrm{rms}}}{E_{0} L_{\mathrm{b}}}\left(\zeta_{x}, \zeta_{x}^{\prime}\right)
$$

where $L_{\mathrm{b}}$ is a bending path length and $\Delta E_{\mathrm{rms}}$ is CSRinduced energy spread after the bending path, $E_{0}$ is reference energy.

As we have seen, the emittance growth depends on both beam transport design and bunch parameters. The CSR wake dispersion is a characteristic function of beam transport design, and obtained by matrix analysis. The energy spread caused by CSR is an intrinsic parameter for the electron bunch which contributes to the emittance growth. The energy spread due to the CSR for a Gaussian bunch is given by

$\frac{\Delta E_{\text {rms }}}{E_{0}}=0.2459 \frac{r_{\mathrm{e}} Q L_{\mathrm{b}}}{e \gamma \rho^{2 / 3} \sigma_{s}^{4 / 3}}$

where $r_{\mathrm{e}}$ is the classical electron radius, $Q$ is bunch charge, $\sigma_{s}$ is RMS bunch length.

The energy spread has a smaller value under vacuum pipe shielding. The shielding effect is evaluated analytically for two parallel conducting plates configuration [39], and given by a handy formula:

$$
\begin{aligned}
\frac{\Delta E_{\mathrm{rms}}^{\text {Shielded }}}{\Delta E_{\mathrm{rms}}^{\text {Free Space }}}= & 1.76 \eta^{1.24} \exp \left(-1.60 \eta+0.0975 \eta^{2}\right. \\
& \left.-0.00246 \eta^{3}\right)
\end{aligned}
$$

where $\eta=\sqrt{2 / 3}(\pi \rho / h)^{3 / 2}\left(\sigma_{s} / \rho\right)$ is the shielding parameter, $h$ is distance between the plates, and the above formula is valid for $1 \leqslant \eta \leqslant 10$. The emittance growth due to the shielded CSR can be estimated by using the matrix method with Eq. (5.9).

As an example of the first-order matrix analysis, we see emittance compensation through a beam transport for a 3GeV ERL light source consisting of triple-bend achromat (TBA) cells as shown in Fig. 6. The bending radius is $25 \mathrm{~m}$, and the bending angle is $(3+6+3)=12^{\circ}$. The quadrupole magnets inside the cell are tuned so that the TBA cell becomes isochronous.

We assume the electron bunch parameters as follows: bunch charge $Q=770 \mathrm{pC}$, bunch length $\sigma_{s}=30 \mu \mathrm{m}$, normalized rms emittance $\varepsilon_{n \text {,rms }}=0.1 \pi \mathrm{mm}$-mrad, uncorrelated energy spread $\sigma_{E} / E_{0}=0.02 \%$, and Gaussian distribution of electrons in the $6 \mathrm{D}$ phase space of motion.

The CSR-induced emittance growth through an ERL loop can be compensated by setting cell-to-cell betatron phase advance at an appropriate value [40]. This emittance compensation can be applied to ERL light sources. We choose the quadrupole parameters of the TBA cell in the 3$\mathrm{GeV}$ ERL so that the betatron phase advance at each cell becomes $\Delta \psi_{x}=\frac{8}{3} \pi$ with keeping isochronous condition. With this design of TBA cells, the CSR kick applied to each bunch slices is expected to be canceled every three-cells. Fig. 7 shows the beam envelopes in three TBA-cells for

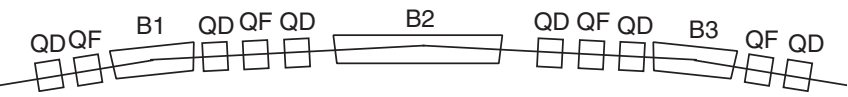

Fig. 6. A triple bend achromatic cell: $\rho=25 \mathrm{~m}, \theta=3+6+3=12^{\circ}$.

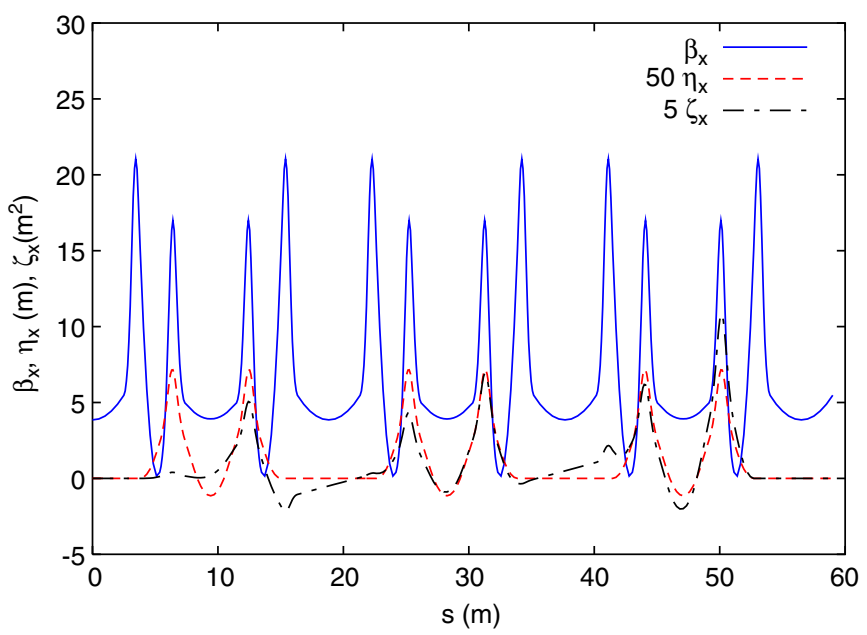

Fig. 7. Beam envelopes for 3 cells, where the betatron phase advance is $\Delta \psi_{x}=\left(\frac{8}{3}\right) \pi$. 


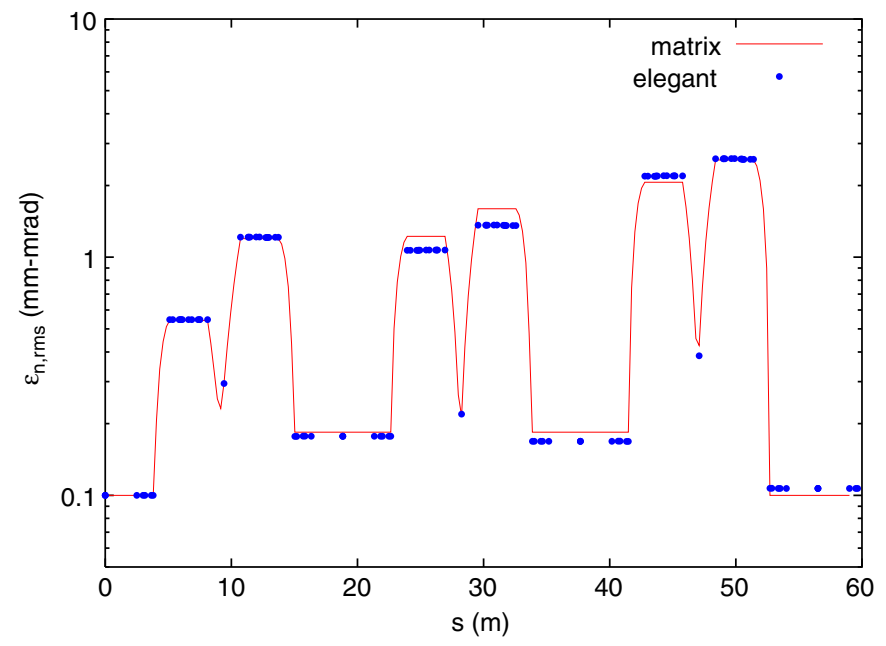

Fig. 8. Emittance along the beam path calculated by matrix and elegant.

$\Delta \psi_{x}=\frac{8}{3} \pi$. We can see both the momentum dispersion and the CSR dispersion disappear after the third cell: $\eta_{x}=\eta_{x}^{\prime}=0, \zeta_{x}=\zeta_{x}^{\prime}=0$. The emittance compensation by cell-to-cell phase matching is confirmed by matrix analysis and a particle tracking code elegant [41]. Fig. 8 shows the calculated emittance along the designed TBA cells. It is clearly seen that the emittance compensation is achieved at the third cell exit.

\subsection{Acknowledgment}

R.H. acknowledges JPPS-15360507 for financial support.

\section{The code $\mathrm{TraFiC}^{4}$ - A. Kabel}

The re-discovery of CSR in the context of its possible detrimental effects in accelerator components with high peak currents and small bending radii, such as magnetic bunch compressors, and wigglers motivated the development of the code $\mathrm{TraFiC}^{4}$ [42-53]; it simulates CSR effects from first principles and is aimed at simulating the macroscopic effects (such as projected emittance growth) in general beamlines and bunch shapes.

\subsection{The core algorithm}

$\mathrm{TraFiC}^{4}$ models the self-interaction of the bunch by storing away the complete history of a bunch of macroparticles as it travels along the beamline. The bunch is specified at a time $t=0$; for all instances $t<0$, the bunch is assumed to have traveled an infinitely extending driftspace. The history of the macroparticles comprising the bunch is described by their centroids' laboratory coordinates and velocities, expressed in terms of $\mathbf{x}, \vec{\beta} / \beta$, and $\beta \gamma$. These are stored at discrete times $t_{1}, \ldots, t_{n}$. For $t_{i} \leqslant t \leqslant t_{i+1}$, the particle is assumed to move only under the influence of the external magnetic lattice. This allows one to find the particle's coordinates for any given $t$ by a quick binary search in the list $t_{1}, \ldots, t_{n}$ and a tracking step. In particular, this history lookup mechanism allows to find, for a given particle trajectory and given observation point and time, the associated retarded time. $\mathrm{TraFiC}^{4}$ uses extended ('smeared') macro-particles with Gaussian profiles whose centroids are tracked, electric and magnetic fields at any given point can be calculated by integrating over their profile over a retarded time interval. As the fields calculated from retarded potentials solve Maxwell's equations exactly, the method should depict all charge-related phenomena, such as longitudinal and transverse space charge and acceleration-generated radiation, provided the temporal and spatial resolution is high enough.

To determine the motion of the bunch under the influence of its own fields, the fields are calculated at times $t_{i+1 / 2}=\left(t_{i}+t_{i+1}\right) / 2$ and positions $\mathbf{x}\left(t_{i+1 / 2}\right)$, the associated momentum kick is applied in half at $t_{i}, \mathbf{x}\left(t_{i}\right)$ and $t_{i+1}, \mathbf{x}\left(t_{i+1}\right)$, changing the six-dimensional initial conditions stored at the times $t_{i}, t_{i+1}$. The momentum kick on each particle originates from the sum of the fields of all macroparticles, making $\mathrm{TraFiC}^{4} O\left(N^{2}\right)$ in the number of macroparticles.

The perturbation of the bunch trajectories may lead to significant changes of the bunch shape and thus the resultant CSR fields at later times; a 'self-consistent' algorithm is necessary to model this behavior. Some other codes and earlier versions of $\mathrm{TraFiC}^{4}$ only could model a 'perturbative' behavior, i.e. the behavior of a bunch under the influence of the fields generated by its unperturbed trajectories. The current version of $\mathrm{TraFiC}^{4}$ has twoalgorithms for self-consistent calculations: (1) A "pong" algorithm, in which two initially identical copies of the generating bunch correct their trajectories by applying the field of the respective other bunch. This mutual correction is repeated a user-defined number of times for each timestep. The deviation of the copies' trajectories give a rough estimate of the error made in the process, (2) a selfcorrecting algorithm, in which all fields of a bunch on itself in a given timestep are collected and subsequently applied at once to all particles. The trajectories are then corrected up to the end of the beamline, and the process repeats (again, the number of iterations is user-defined) or advances to the next timestep.

\subsection{Bunch setup}

TraFiC ${ }^{4}$ knows three kinds of bunches: generating bunches, comprising weighted, smoothed-out macro particles which generate the fields and may or may not move under the influence of their own fields, depending on whether or not the user expects these effects to be important; optical bunches, which do not generate fields, but feel the perturbations of the fields caused by the generating bunch(es), and sampling bunches, which sample the fields of the generating bunches, but are not influenced 
by them (they can be viewed as co-moving ObserverGrids), see below).

Generating bunches consist of one-, two-, or threedimensional extended Gaussian charge distributions whose centroids are tracked according to the magnetic lattice and possibly the bunch's fields. All other bunches comprise point particles, which can bear a charge (or statistical weight), which will be used in calculating collective quantities. Sampling and optical bunches do not contribute to the fields, they can be used to study the behavior of subensembles of the bunch.

A bunch in $\mathrm{TraFiC}^{4}$ is set up by specifying a particle class (point, pencil, sheet, or cylinder) and a Generator. A Generator is a sequence of extended phasespace vectors (extended meaning 6 phasespace coordinates +1 statistical weight); pre-defined generators are Cartesian Grids, quasirandom sequences, and input from a text file. Generators can be transformed into new Generators using a set of predefined functions (transform to beam ellipses with given Twiss parameters, Scale, Shift, transform to Gaussian normal distribution), Selectors (first $n$ particles from a generator, particles lying on either side of a hyperplane), and arbitrary user-defined functions. All transformations can be concatenated, allowing for high flexibility in populating the bunch.

\subsection{Lab and local frames}

$\mathrm{TraFiC}^{4}$ is able to handle fully three-dimensional problems; there is no limitation to one plane of movement. While $\mathrm{TraFiC}^{4}$ does all of its tracking in Cartesian laboratory coordinates, as it needs to store the history of all particles to calculate the retarded fields, as a local system, a Frenet coordinate system associated with an 'orbit' particle's trajectory $\vec{r}_{0}(s)$ is used. The co-moving frame is spanned by $\vec{r}_{0}^{\prime}(s), \vec{r}_{0}^{\prime \prime}(s)$, and $\vec{r}_{0}^{\prime}(s) \times \vec{r}_{0}{ }^{\prime}(s)$; the associated normalized vectors are $\vec{t}(s), \vec{n}(s)$, and $\vec{b}(s)$. Then, we find that particle's local coordinates $x, y, l$ by $\vec{r}(s(t))=x \vec{n}(s(t)+l)+y \vec{b}(s(t)+l)$, which agrees with the usual accelerator coordinates in beamlines with curvature of constant direction. All collective quantities are calculated in terms of these local coordinates, their first derivative with respect to $s$, and the energy deviation; also, all quantities are output in a version with dispersion subtracted.

\subsection{Beamline and elements}

Beamlines can be set up as a sequence of drift spaces, dipole bending magnets, extended (thick) quadrupoles, and thin sextupoles. Also, there are some zero-length 'control' elements (see below).

Bending can be rotated around the axis of the incoming particle by an arbitrary angle. This makes $\mathrm{TraFiC}^{4}$ fully three-dimensional, as the movement of the orbit particle is not restricted to a plane any more. A dipole can be tilted upward and downward, i.e., rotated around the curvature vector. This turns the trajectory of the orbit from a circular arc into a helical segment. The magnet's edge faces may be rotated away from a sector-bend setup; the transverse effects are modeled by sandwiching the dipole between thin-lens focusing elements, while the radial ones follow from correct geometric tracking in the lab frame.

\subsection{Observer grids}

Sometimes it is of interest to calculate the fields due to CSR not only within the bunch, but at observation points far away from the bunch, such as for the calculation of coherent radiation at detectors or targets. $\mathrm{TraFiC}^{4}$ now allows to specify such observation points. It is most convenient to do that with. An artificial beam-line element type ObserverGrid allows to specify an observer with reference to the beam-line coordinates. By specifying numbers $n_{t}, t_{\min }, t_{\max } ; n_{s}, s_{\min }, s_{\max } ; n_{x}, x_{\min }, x_{\max }$; $n_{y}, y_{\min }, y_{\max } ; \beta_{s}, \beta_{x}, \beta_{y}$, a four-dimensional lattice of size $n_{t}, n_{s}, n_{x}, n_{y}$, spanning the spacetime interval $\left[t_{\min }, t_{\max }\right] \otimes\left[s_{\min }, s_{\max }\right] \otimes\left[x_{\min }, x_{\max }\right] \otimes\left[y_{\min }, y_{\max }\right]$, where $t$ refers to the laboratory time and $s, x, y$ refer to the local tangential, radial, and transverse direction, resp., is created. The observation grid moves with a speed of $c \vec{\beta}$. The quantities calculated are the total force per charge $\vec{E}+$ $\vec{\beta} \times \vec{B}$ and the energy change per charge $\vec{E} \cdot \vec{\beta}$. An arbitrary number of ObserverGrids can be defined.

\subsection{Parallelization and load balancing}

The $O\left(N^{2}\right)$ runtime behavior makes a parallel version of $\mathrm{TraFiC}^{4}$ a necessity for all but the simplest calculation. Parallelization is achieved by a message-passing mechanism: replicated versions of $\mathrm{TraFiC}^{4}$ run on different processors, calculating momentum kicks on disjoint subsets of particles and/or observation points. In an all-to-all synchronization step, the momentum kick information is updated between processors, and the calculation continues independently for the next time step. $\mathrm{TraFiC}^{4}$ allows for the automatic distribution of particles to processors according to the measured speeds in previous timesteps. The load balancer will react to changes in speed according to a user-selectable inertia. Moreover, the load balancer associated with each bunch and ObserverGrid can learn from the speed behavior of calculations for bunches treated before, distributing particles accordingly.

\subsection{Documentation and source code}

$\mathrm{TraFiC}^{4}$ has a rudimentary user's manual [54]. Also, the class structure of the tracking part has been extensively documented, which should make it possible for programmers to add their own element types or transformer functions [55].

The source code for $\mathrm{TraFiC}^{4}$ is available from Ref. [56]. $\mathrm{TraFiC}^{4}$ requires a recent, ANSI-compliant $\mathrm{C}++$ compiler, a Fortran 77 compiler, some components of the 


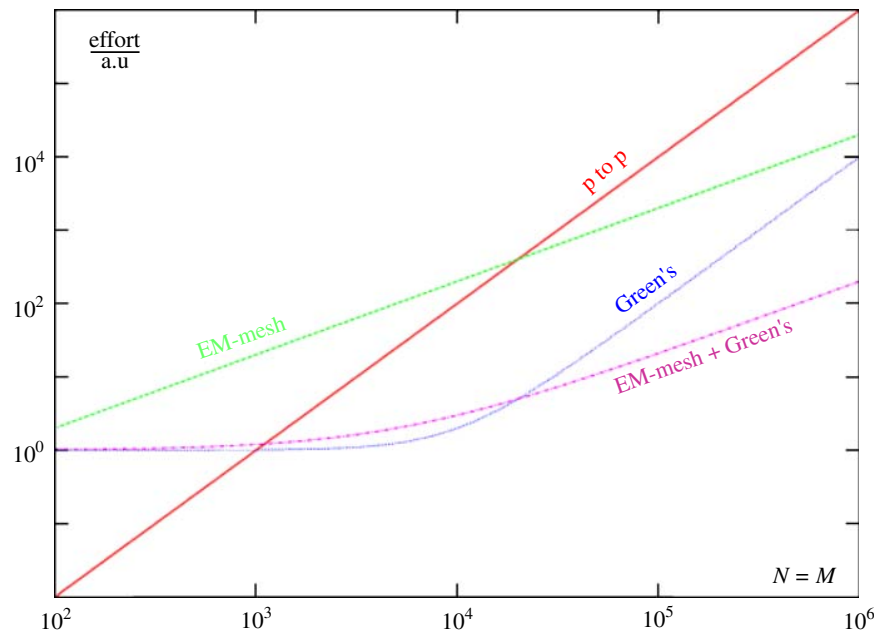

Fig. 9. Numerical effort of different CSR field calculation methods vs. \# of tracked particles.

"boost" extension library [57], and an MPI multiprocessing library.

\section{Efficient methods for CSR calculations in CSRtrack-M. Dohlus and T. Limberg}

CSRtrack is a new code for the simulation of Coherent Synchrotron Radiation effects on the beam dynamics of linear accelerators. It incorporates the physics of our previous code, $\mathrm{TraFiC}^{4}$ [58], and adds new algorithms for the calculation of the CSR fields. A one-dimensional projected method allows quick estimates and a Green's function method allows 3-D calculations about hundred times faster than with the direct method for large particle numbers. Tracking for sub-bunch numbers of $>10^{5}$ is made possible by combining the Green's function algorithm with a meshed fields method, as we demonstrate with an example below.

\subsection{Introduction}

Essentially two types of approaches are presently used for the self-consistent calculation of particle distributions on curved trajectories. Both are available in CSRtrack.

The 1-D approach uses a simplified model for the calculation of longitudinal forces [61]. It neglects transverse forces as well as transverse beam dimensions and assumes that the longitudinal distribution is unchanged at retarded times. A renormalized Coulomb term is used to extract the field singularity in the 1-D beam.

The sub-bunch approach uses a set of 3-D charge distributions, ${ }^{1}$ e.g., time-independent Gaussian, to approximate the source distribution. The physical model of the sub-bunch method is complete, but the resolution of phase space modeling is severely limited by the numerical effort for the field calculation of all point to point interactions.
Even with parallel computing particle numbers above $10^{3}$ are difficult to handle (CPU time on a cluster is many days).

TraFiC ${ }^{4}$ and CSRtrack ease that problem by using a convolution method [49] to reduce the field calculation of 3$\mathrm{D}$ sources to 1-D integrations (for each interaction), gaining about a factor of ten in manageable particle numbers.

Further improvement is possible with a pseudo Green's function approach and the calculation of electromagnetic fields on a mesh. The pseudo Green's function uses the discretized field of one reference sub-bunch to calculate all interactions. The disadvantage that the effort scales quadratic with the number of particles can be avoided by calculating the electromagnetic fields on a mesh. With a combination of mesh field calculation and pseudo Green's function method, tracking calculations with $>10^{5}$ particles in less then one day (using 20 CPUs in parallel) have been done.

\subsection{Numerical aspects of different CSR field calculation methods}

In the following we discuss different algorithms and their relative computation time consumption, which are summarized in Fig. 9.

A word of warning: our discussion is focused to the aspects of efficient field calculation but not to the control of the width of track steps. Under some circumstances (e.g. if the initial bunch density is modulated or bunch compressors with shielding) the difference of the group velocity of electromagnetic waves and the motion of the bunch needs an integration with rather short steps to avoid significant errors due to undersampling.

\subsubsection{One-dimensional approach-projected method}

The particle distribution is projected to the reference trajectory and a smooth one-dimensional charge density $\lambda(s)$ is calculated by binning or filtering. The smoothing is crucial for the stability and accuracy of the simulation because the microbunch instability is sensitive to high frequency components in the charge density.

The longitudinal field $E^{(\lambda)}$ can be calculated by onedimensional integration

$E^{(\lambda)}\left(s_{0}, t_{0}\right)=\int \lambda^{\prime}\left(u+s_{0}-v t_{0}\right) K\left(s_{0}, u\right) \mathrm{d} u$,

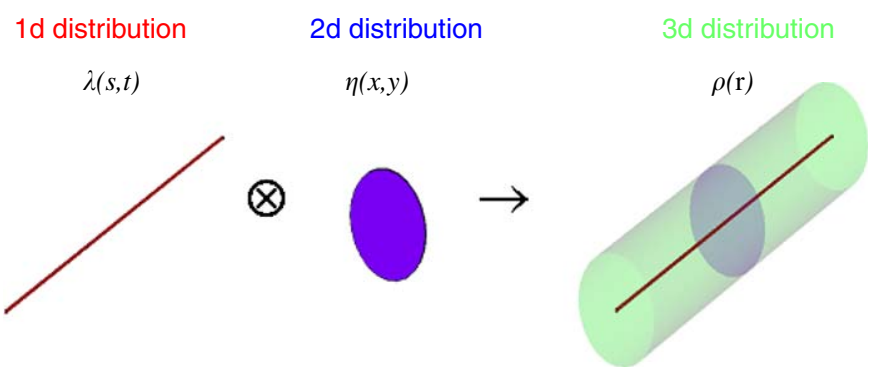

Fig. 10. Schematic of the convolution method.

\footnotetext{
12-D sub-bunches in R.Li's code [59].
} 
with the kernel function $K\left(s_{0}, u\right)$ that depends on the geometry of the trajectory. This integral is a convolution in time. It can be solved efficiently by FFT methods if $E^{(\lambda)}$ is required for a time interval.

The longitudinal field is calculated on a mesh and interpolated to the projected particle positions. The particle tracking takes into account external fields and the longitudinal self field. The effort for the field calculation depends linearly on the number of particles.

\subsubsection{Sub-bunch approach}

The distribution of source particles is described by a set of sub-bunches with well defined shape. The individual trajectory of each sub-bunch has to be known in absolute coordinates back in time. This defines the 3-D source distribution $\rho(\mathbf{r}, t)$ so that potentials and electromagnetic fields can be calculated by an integration of retarded sources.

A disadvantage of the sub-bunch approach is the large numerical effort. For $M$ test particles and $N$ source distributions (sub-bunches) $M \times N$ three-dimensional integrations have to be performed for every time step.

\subsubsection{Source distributions and test particles}

For self consistent tracking the trajectory of each source is defined by the motion of an associated test particle. Therefore, $M=N+M_{t}$ test particles are needed, with $M_{t}$ the number of additional particles that can be used for more detailed explorations e.g. of the transverse phase space. The effort for self consistent tracking is at least proportional to $N^{2}$. Additional test particles need the computation of $N \times M_{t}$ interactions.

If the bunch shape is weakly deformed due to self forces a perturbation approach can be used. The self forces to the test particles are calculated for unperturbed motion of source distributions and test particles. These forces are used in a second step to calculate the perturbed motion. The initial condition of test particles can be set as required (e.g. to investigate an individual slice) but at least some test particles should be used to verify the perturbation approach.

\subsubsection{3-D sub-bunches, convolution method}

Since the field of each source sub-bunch has to be calculated for every test position by a 3-D integration of retarded sources, the full field computations is quite time consuming. For spherical Gaussian sub-bunches this integration can be reduced to a 1-D integral [62].

The codes $\mathrm{TraFiC}^{4}$ and CSRtrack adopt a calculation method that was developed in Ref. [49]. A certain type of 3$\mathrm{D}$ distribution can be represented by the convolution of a longitudinal 1-D profile $\lambda(s, t)$ with a transverse 2-D density function $\eta(x, y)$ (Fig. 10). Then the 3-D electromagnetic fields can also be calculated by a convolution of fields $\mathbf{E}^{(\lambda)}$, $\mathbf{B}^{(\lambda)}$ caused by the 1-D source with the 2-D density function. $\mathbf{E}^{(\lambda)}$ and $\mathbf{B}^{(\lambda)}$ are split into singular parts $\mathbf{E}^{(\lambda, s)}$ and $\mathbf{B}^{(\lambda, s)}$, which are dominated by local effects, and nonsingular parts $\mathbf{E}^{(\lambda, n s)}$ and $\mathbf{B}^{(\lambda, n s)}$. The asymptotic behavior of the singular part is analytically known so that its convolution with certain transverse density functions can be computed efficiently.

As the nonsingular parts depend mainly on long range interactions, their transverse dependence is weak and the convolutions $\mathbf{E}^{(\lambda, n s)} \otimes \eta, \mathbf{B}^{(\lambda, n s)} \otimes \eta$ can be approximated by $\mathbf{E}^{(\lambda, n s)}$ and $\mathbf{B}^{(\lambda, n s)}$ for sufficiently small transverse dimensions ( $\ll\left(R_{0} \sigma_{\lambda}^{2}\right)^{1 / 3}$, with $R_{0}$ the curvature radius and $\sigma_{\lambda}$ the bunch length). Therefore, the numerical effort $E_{c}$ is determined by the 1-D integration for the nonsingular parts. The total effort for all source to test-point interactions is $M \times N \times E_{c}$.

\subsubsection{Pseudo Green's function approach}

The fields $\mathbf{E}^{(0)}\left(\mathbf{r}, t_{0}\right), \mathbf{B}^{(0)}\left(\mathbf{r}, t_{0}\right)$ of a reference sub-bunch (charge $q_{0}$ ) that travels along a reference trajectory $\mathbf{r}_{0}(t)$ are used to approximate the fields of other sub-bunches (charge $q_{v}$ ), trajectory $\mathbf{r}_{v}(t)$. This approach neglects vertical forces. At the observation time $t_{0}$ the trajectory of subbunch $v$ is approximated by

$\mathbf{r}_{v} \approx \mathbf{r}_{v}\left(t_{0}\right)+\mathbf{R} \cdot\left(\mathbf{r}_{0}(t)-\mathbf{r}_{0}\left(t_{0}\right)\right)$.

This shift-rotation-transformation (with rotation operator $\mathbf{R})$ is used to calculate

$\mathbf{E}^{(v)}\left(\mathbf{r}, t_{0}\right) \approx \frac{q_{v}}{q_{0}} \mathbf{R} \cdot \mathbf{E}^{(0)}\left(\mathbf{r}_{0}\left(t_{0}\right)+\mathbf{R}^{-1}\left(\mathbf{r}-\mathbf{r}_{v}\left(t_{0}\right)\right), t_{0}\right)$

and $\mathbf{B}^{(v)}\left(\mathbf{r}, t_{0}\right)$ in the same way. The pseudo Green's functions $\mathbf{E}^{(0)}\left(\mathbf{r}, t_{0}\right), \mathbf{B}^{(0)}\left(\mathbf{r}, t_{0}\right)$ are calculated once for every time step on a 2-D mesh in the horizontal plane with $M_{g}$ points:

$\mathbf{E}^{(0)}\left(\mathbf{r}, t_{0}\right)=E_{x}(x, y) \mathbf{u}_{x}+E_{y}(x, y) \mathbf{u}_{y}$

$\mathbf{B}^{(0)}\left(\mathbf{r}, t_{0}\right)=B_{z}(x, y) \mathbf{u}_{z}$.

The numerical effort for the calculation of all self forces is $M_{g} \times E_{c}+M \times N \times E_{i, g}$ with $E_{i, g}$ the effort for the interpolation on the mesh. The approach is effective for $N \times M>M_{g}$. Simulations with many particles $\left(>10^{4}\right)$ are not limited by the effort for the field calculation $\left(M_{g} \times E_{c}\right)$ but by the effort for the interpolation $\left(M \times N \times E_{i, g}\right)$.

\subsubsection{Meshed EM fields}

If the density of particles is large compared to the fine structure of the particle distribution (and the self-fields) it is more efficient to calculate electromagnetic fields on a mesh with $M_{e m}$ points and to interpolate them to the test points. The numerical effort is $M_{e m} \times N \times E_{c}+M \times E_{i, e m}$ with $E_{i, e m}$ the effort for the interpolation on the field mesh. The total effort is no longer proportional to the squared number of particles ( $M \times N$ with $M \geqslant N$ for self consistent tracking)!

Reasonable EM meshes need at least $M_{e m}>10^{4}$ points and therefore the method gets efficient for $M, N>10^{4}$. The effort for such particle numbers is still too large (even with parallel computing) for routine investigations. 
This is different if the meshed fields approach is combined with the pseudo Green's function approach: the total effort is $M_{g} \times E_{c}+M_{e m} \times N \times E_{i, g}+M \times E_{i, e m}$.

\subsubsection{Iterative tracking}

For tracking, self-forces have to be calculated for particles on phase space coordinates that depend on the unknown forces. If the track step is sufficiently small the forces can be approximated for phase space positions calculated without self contributions. To avoid the numerical effort for very many small steps iterative tracking with medium step size is used: estimated self forces are calculated for estimated particle positions and are used to improve the position estimation. The iteration is repeated until an error criterion is fulfilled.

The numerical integration of the equation of motion in CSRtrack uses sub-steps that are (much) smaller than the steps of the iterative force calculation. The forces at substeps are linearly interpolated between the force at the start point of an iterative step and at its end point. The error due to this linear interpolation and due to too big iterative steps is not reduced by iterative tracking.

CSRtrack provides two possibilities to determine the size of iterative steps: by a recursive algorithm that is based on the bunch length and curvature radius of the trajectory and by external definition. Like in all self-field applying tracking codes, the result has to some extent to be interpreted and justified by the user (e.g. convergence tests or the inspection of the forces observed by some selected test particles).

\subsection{CSRtrack features}

\subsubsection{Particles definition}

Position, momentum and charge of each particle of the distribution are defined in an absolute Cartesian coordinate system with the horizontal plane as $x y$-plane. Particles with nonzero charge are source particles that create selffields that affect the motion of test particles. In the present versions all particles have the source and test property.

\subsubsection{Lattice}

CSRtrack supports magnetic dipole- and multipole-fields that are defined in Cartesian coordinates. The range of the fields of one element is limited by two field boundaries perpendicular to the horizontal plane. The dipole field between two field boundaries is constant and parallel to the vertical $z$-axis. Together with the dipoles a reference trajectory is defined that consists of arcs and lines.

To take into account vertical edge focusing at the hard edges of dipoles the tracking algorithm applies discrete kicks proportional to the vertical offset. The magnetic multipole field is defined in a local coordinate system (longitudinal, horizontal, vertical) with its origin in the intersection point of the reference trajectory and the field boundary.
The magnetic multipole field between field boundaries depends only on transverse coordinates. It is characterized by its strength, azimuthal order, transverse offset and skew angle.

\subsubsection{Shielding}

CSRtrack provides shielding as of now with parallel ideal conducting plates with constant distance to the beam. The user has to specify the maximum distance to the beam up to which mirror charges will be taken into account.

\subsubsection{Parallel computing}

CSRtrack is available as a parallel processing code using the MPI protocol with the MPICH package. The fast 1-D method is not parallel processing.

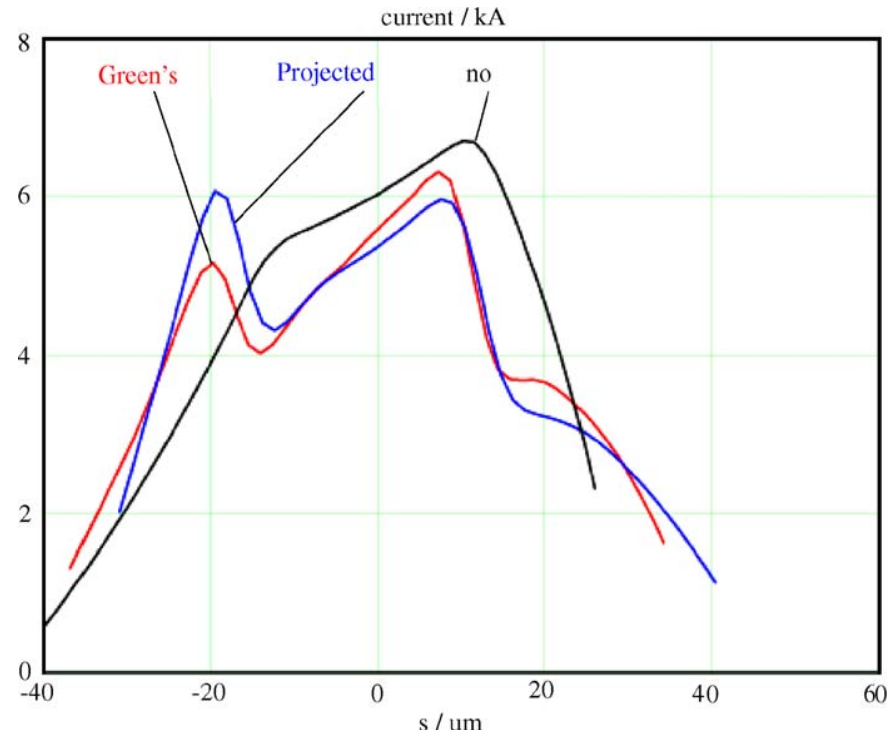

Fig. 11. Bunch current vs. longitudinal position. Green's: Green's function method, Projected: 1-D method, no: no CSR fields.

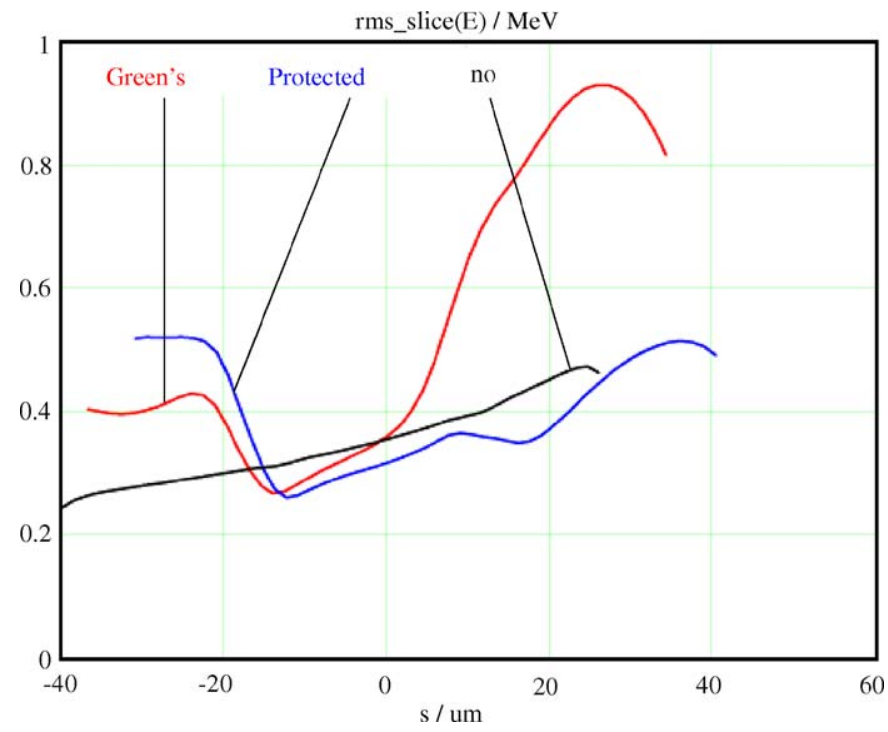

Fig. 12. Uncorrelated energy spread (RMS) vs. longitudinal bunch position. 


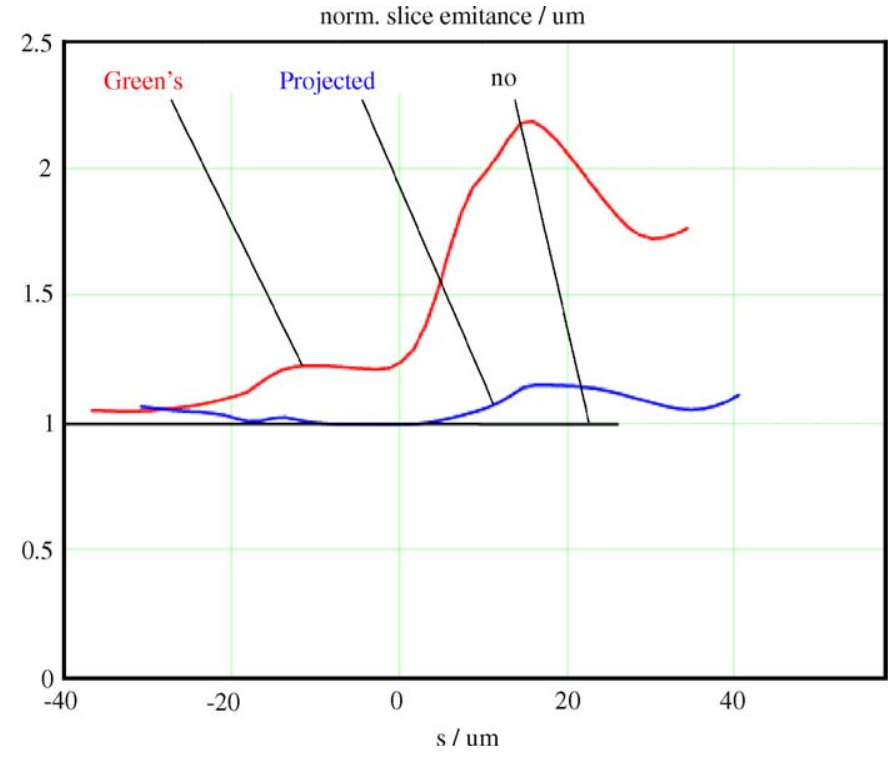

Fig. 13. Normalized horizontal slice emittance vs. longitudinal bunch position.

Table 1

Comparison of some results and the numerical effort. The double numbers in the lower rows refer to the two peaks of the distributions

\begin{tabular}{lll}
\hline Method & Projected (1d) & Green's funct. \\
\hline $\begin{array}{l}\text { RMS sub-bunch dimensions: } \\
\text { Longitudinal } / \mu \mathrm{m}\end{array}$ & 2 & 2 \\
Horizontal $/ \mu \mathrm{m}$ & & 34 \\
Vertical $/ \mu \mathrm{m}$ & & 100 \\
Projected emittance ${ }^{*} \gamma / \mu \mathrm{m}$ & 3.81 & 6.93 \\
$s_{\text {peak }} / \mu \mathrm{m}$ & $-19.5 \mid 7.5$ & $-19.8 \mid 7.2$ \\
$I_{\text {peak }} / \mathrm{kA}$ & $6.09 \mid 5.98$ & $5.16 \mid 6.30$ \\
Slice emittance ${ }^{*} \gamma / \mu \mathrm{m}$ & $1.02 \mid 1.04$ & $1.11 \mid 1.83$ \\
Slice energy spread $/ \mathrm{MeV}$ & $0.46 \mid 0.36$ & $0.38 \mid 0.54$ \\
CPU time $(3 \mathrm{GHz}, 64 \mathrm{bit})$ & 9 min $(1 \mathrm{CPU})$ & $17 \mathrm{~h}(20 \mathrm{CPUs})$ \\
\hline
\end{tabular}

\subsection{Comparing results for different methods}

For the calculations in this chapter, we use the "Zeuthen chicane"; a benchmark case (available on the web), where a bunch of $1 \mathrm{nC}$ charge is compressed to reach a peak current of $6 \mathrm{kA}$ at a beam energy of $500 \mathrm{MeV}$ [22]. A different incoming bunch shape than in Ref. [22] is used: a trapezoid with a peak current of $600 \mathrm{~A}$, with $250 \mu \mathrm{m}$ long ramps and a $250 \mu \mathrm{m}$ long flat top. The uncorrelated energy spread is $36 \mathrm{keV}$.

174600 sub-bunches in 360 longitudinal slices are used to model the bunch. The regular slice distribution is composed by 97 different points for the transverse phase space times 5 values for the uncorrelated energy spread.

Figs. 11-13 show results at a point two meters downstream of the last chicane dipole magnet. The red line shows the Green's function method result, the blue that of the 1-D method and the black line the result without CSR fields. Some numbers are summarized in Table 1. It has to be emphasized that the numerical effort for the $1 \mathrm{~d}$ method is quite small compared to that for the Green's function method, but especially the growth of the slice emittance is significantly underestimated. The $1 \mathrm{~d}$ method predicts a growth of few \% in the core of the bunch while the Green's approach obtains between 20 and 100\%

\section{References}

[1] LCLS Design Study Report, SLAC, 1998; TESLA X-ray FEL project, see $\langle$ http://tesla-new.desy.de〉.

[2] For an overview, see Energy Recovery Linacs Light Sources: an Overview, J. B. Murphy, Proceedings of the 2003 Particle Accelerator Conference, p. 176, paper TOAC001.

[3] M. Borland, et al., Start-to-End Jitter Simulations of the Linac Coherent Light Source, Proceedings of the 2001 PAC 2707 (2001).

[4] G. Stupakov, R. Warnock, Microbunch instability theory and simulations, ICFA Beam Dynamics Newsletter, No. 35, December 2004.

[5] S. Heifets, G. Stupakov, S. Krinsky, Phys. Rev. ST Accel. Beams 5 (2002) 064401.

[6] Z. Huang, K. Kim, Phys. Rev. ST Accel. Beams 5 (2002) 074401.

[7] Z. Huang, M. Borland, P. Emma, K.-J. Kim, Nucl. Instr. and Meth. A 507 (2003) 318.

[8] ICFA Beam Dynamics Newsletter, No. 35, December 2004.

[9] B.S. Schmekel, R.V.E. Lovelace, I.M. Wasserman, Phys. Rev. E 71 (2005) 046502.

[10] J.B. Murphy, An Introduction to Coherent Synchrotron Radiation in Storage Rings, ICFA Beam Dynamics Newsletter, No. 35, December 2004.

[11] G. Bassi, Note on the Full Fourier Method for the Field Calculation of Coherent Synchrotron Radiation from Arbitrary Planar Orbits, available at $\langle$ http: / / www. math. unm. edu/ gbassi $\rangle$.

[12] M. Borland, Elegant: a flexible SDDS-compliant code for accelerator simulation, Advanced Photon Source LS-287, September 2000.

[13] M. Borland, Phys. Rev. ST Accel. Beams 4 (2001) 070701.

[14] E.L. Saldin, E.A. Schneidmiller, M.V. Yurkov, Nucl. Instr. and Meth. A 398 (1997) 373.

[15] G. Stupakov, P. Emma, CSR wake for a short magnet in ultrarelativistic limit, Proceedings of EPAC 2002, Paris, France.

[16] M. Borland, Coherent synchrotron radiation and microbunching in bunch compressors, Proceedings of LINAC 2002, Gyeongju, Korea.

[17] L. Bentson, P. Emma, P. Krejcek, A new bunch compressor chicane for the SLAC linac to produce $30-\mathrm{Fsec}, 30-\mathrm{KA}, 30-\mathrm{GeV}$ electron bunches, Proceedings of EPAC 2002, Paris, France.

[18] R. Li, Nucl. Instr. and Meth. A 429 (1998) 310.

[19] M. Venturini, R. Warnock, R. Ruth, J.A. Ellison, Impedance description of coherent synchrotron radiation with account of bunch deformation, Phys. Rev. ST Accel. Beams 8 (2005) 014202.

[20] M. Venturini, R. Warnock, R. Ruth, J.A. Ellison, Coherent synchrotron radiation and bunch stability in a compact storage ring, Phys. Rev. ST Accel. Beams 8 (2005) 014202.

[21] M. Venturini, R. Warnock, Phys. Rev. Lett. 89 (2002) 224802.

[22] ICFA Beam Dynamics Mini-Workshop on CSR, Berlin-Zeuthen, 2002, 〈http://www. desy.de/csr $\rangle$.

[23] G.V. Stupakov, I.A. Kotelnikov, Phys. Rev. ST Accel. Beams 6 (2003) 034401.

[24] T. Agoh, K. Yokoya, Phys. Rev. ST Accel. Beams 7 (2004) 054403.

[25] M. Bassetti, G.A. Erskine, Closed expression for the electrical field of a two-dimensional Gaussian charge, CERN Report No. CERN-ISR$\mathrm{TH} / 80-06,1980$.

[26] R. Warnock, G. Bassi, J.A. Ellison, Vlasov treatment of coherent synchrotron radiation from arbitrary planar orbits, Proceedings of ICAP2004, St. Petersburg, Russia, Nucl. Instr. and Meth. A, in press, doi: 10.1016/j.nima.2005.11.070. 
[27] G. Bassi, J.A. Ellison, R. Warnock, Progress on a Vlasov treatment of coherent synchrotron radiation from arbitrary planar orbits, Proceedings of PAC2005, Knoxville, TN, USA.

[28] S. Heifets, G. Stupakov, S. Krinsky, Phys. Rev. ST Accel. Beams 5 (2002) 064401.

[29] L. Serafini, Computational modeling of high-brightness electron beam physics, in: J. Rosenzweig, L. Serafini (Eds.), The Physics of High Brightness Beams, World Scientific, Singapore, ISBN 981-024422-3, June 2000.

[30] L. Giannessi, M. Quattromini, Phys. Rev. ST Accel. Beams 6 (2003) 120101; 〈http://www.tredi.enea.it) and /http://projects. astec.ac.uk/Plone/eurofel/codes/spacecharge/tredi//.

[31] L. Giannessi, M. Quattromini, C. Ronsivalle, Emittance dilution due to 3D perturbations in RF-photoinjectors, Proceedings of the Ninth European Particle Accelerator Conference, July 5-9, 2004, Lucerne, Switzerland, available at $\langle$ http: //www. jacow.org $\rangle$;

L. Giannessi, M. Quattromini, C. Ronsivalle, Spectral analysis of charge emission spatial inhomogeneities and emittance dilution in RF guns, Proceedings of the 26th International FEL Conference, August 29-September 3, 2004 Trieste, Italy, also available at (http:// www. jacow. org $\rangle$.

[32] See e.g. 〈http://laacg1.lanl.gov/laacg/services/ parmela.html).

[33] Limborg, et al., Code comparison for simulations of photo-injectors, Proceedings of the 2003 Particle Accelerator Conference, May 12-16, 2003, Portland, Oregon, 〈http: / /www. jacow. org $\rangle$.

[34] L. Giannessi, M. Quattromini, TREDI Reference Manual, See 〈http://www.tredi.enea.it), in preparation.

[35] L. Giannessi, P. Musumeci, M. Quattromini, Nucl. Instr. and Meth. A 436 (1999) 443.

[36] J.B. Rosenzweig, P. Musumeci, Phys. Rev. E (Rapid Comm.) 58 (1998) R2737.

[37] R. Hajima, Japan J. Appl. Phys. 42 (2003) L974.

[38] R. Hajima, R-matrix analysis of the CSR effect in a future ERL light source, in: Proceedings of the 2004 Asian Particle Accelerator Conference, Gyeongju, Korea, 2004.

[39] R.L. Warnock, Shielded coherent synchrotron radiation and its effect on very short bunches, SLAC-PUB-5375, 1990.

[40] D. Douglas, Suppression and enhancement of CSR-driven emittance degradation in the IR-FEL driver, Thomas Jefferson Natl. Acc. Lab. Tech. Note, JLAB-TN-98-012, 1998;

J.H. Wu, et al., Coherent synchrotron radiation analysis for the photoinjected energy recovery linac and UVFEL projects at the NSLS, Proceedings of the 2001 Particle Accelerator Conference, Chicago, USA, IEEE, New Jersey, 2001, p. 2866.

[41] M. Borland, ELEGANT version 14.8, November 26, 2002; Argonne National Laboratory Advanced Photon Source Report No. LS-287, 2000.

[42] A. Kabel, Coherent synchrotron radiation calculations using $\mathrm{TraFiC}^{4}$ : multi-processor simulations and optics scans, in: Proceedings of the IEEE Particle Accelerator Conference (PAC2001), Chicago, Illinois, 18-22 June, 2001.

[43] M. Borland, H. Braun, S. Doebert, L. Groening, A. Kabel, Recent experiments on the effect of coherent synchrotron radiation on the electron beam of CTF II, in: Proceedings of the IEEE Particle Accelerator Conference (PAC2001), Chicago, Illinois, 18-22 June, 2001.

[44] A. Kabel, M. Dohlus, T. Limberg, Nucl. Instr. and Meth. A 455 (2000) 185

[45] Numerical calculation of coherent synchrotron radiation effects, invited talk given at the International Symposium on New Visions in Laser Beam Interactions: Fundamental Problems and Applications of Laser Compton Scattering, Tokyo, Japan, 11-15 October, 1999.

[46] Coherent Synchrotron Radiation Measurements in the CLIC Test Facility (CTF II), H.H. Braun, R. Corsini, L. Groening, F. Zhou, A. Kabel, T. Raubenheimer, R. Li, T. Limberg. eConf, C000821:TH206, 2000.

[47] H.H. Braun, R. Corsini, L. Groening, F. Zhou, A. Kabel, T. Raubenheimer, R. Li, T. Limberg, Phys. Rev. ST Accel. Beams 3 (2000) 124402 .

[48] M. Dohlus, A. Kabel, T. Limberg, Nucl. Instr. and Meth. A 445 (2000) 338.

[49] M. Dohlus, A. Kabel, T. Limberg, Nucl. Instr. and Meth. A 445 (2000) 84.

[50] A. Kabel, M. Dohlus, T. Limberg, Optimal beam optics in the TTF-FEL bunch compression sections minimizing the emittance growth, Proceedings of the IEEE Particle Accelerator Conference (PAC 99), New York, NY, 29 Mar-2 Apr, 1999.

[51] A. Kabel, M. Dohlus, T. Limberg, Uncorrelated emittance growth in the TTF-FEL bunch compression sections due to coherent synchrotron radiation and space charge effects, in: Proceedings of the 6th European Particle Accelerator Conference EPAC 98, Stockholm, Sweden, 22-26 June, 1998, DESYM-98-0601

[52] M. Dohlus, A. Kabel, T. Limberg, Design consequences of coherent synchrotron radiation beam dynamic effects on the TTF-FEL bunch compression system, in: Proceedings of the 19th International Conference on Free Electron Lasers, Beijing, China, 18-21 August, 1997 DESY-TESLA-FEL-97-06B, October 1997.

[53] M. Dohlus, A. Kabel, T. Limberg, Wake fields of a bunch on a general trajectory due to coherent synchrotron radiation, in: Proceedings of the 17th IEEE Particle Accelerator Conference (PAC 97), Technology and Applications, Vancouver, Canada, 12-16 May, 1997 DESY-M-97-10J.

[54] A. Kabel, A Short Guide to $\mathrm{TraFiC}^{4} 2.0 / \mathrm{TraFiC}^{4} 2.0$ Programmer's Reference Manual, 2003.

[55] A. Kabel, TraFiC ${ }^{4} 2.0$ - Programmer's Manual, 2003.

[56] $\mathrm{TraFiC}^{4}$ homepage.

[57] 〈http: //www. boost.org .

[58] T. Limberg, A. Kabel, M. Dohlus, Nucl. Instr. and Meth. 455 (2000) 185.

[59] R. Li, Self-consistent simulation of the CSR effect, EPAC1998, Stockholm, Sweden, June 1998.

[61] E. Saldin, E. Schneidmiller, M. Yurkov, Nucl. Instr. and Meth. A 417 (1998) 158.

[62] M. Dohlus, Two methods for the calculation of CSR fields, TESLAFEL-2003-05. 